\title{
First Total Synthesis of Gliomasolide $C$ and Formal Total Synthesis of Sch-725674
}

\author{
B. Seetharamsingh, Pankaj V. Khairnar and D. Srinivasa Reddy* \\ CSIR-National Chemical Laboratory, Dr. Homi Bhabha Road, Pune, 411008, India. \\ *Corresponding author: \\ Dr. D. Srinivasa Reddy \\ National Chemical Laboratory (CSIR, India), \\ Division of Organic Chemistry, \\ Dr. Homi Bhabha Road, \\ Pune 411008, Maharashtra, \\ India. \\ E-mail: ds.reddy@ncl.res.in
}

Tel. +912025902445

Fax. +912025902629

\section{Supporting Information}

${ }^{1} \mathrm{H}$ and ${ }^{13} \mathrm{C}$ NMR spectra 
NMR comparisons of Sch-725674: Natural (500 MHz, CD 30 OD), synthetic (400 $\mathrm{MHz}$, $\underline{\mathrm{CD}}_{3} \underline{\mathrm{OD}):}$

\begin{tabular}{|c|c|c|c|}
\hline $\begin{array}{l}{ }^{1} \mathrm{H} \text { NMR (Natural) } \\
\delta_{\mathrm{H}}(\text { mult, } J \text { in } \mathrm{Hz})\end{array}$ & $\begin{array}{l}{ }^{1} \mathrm{H} \text { NMR (synthetic) } \\
\delta_{\mathrm{H}}(\text { mult, } J \text { in } \mathrm{Hz})\end{array}$ & $\begin{array}{l}{ }^{13} \mathrm{C} \text { NMR } \\
\text { (natural) } \\
\delta_{\mathrm{C}}\end{array}$ & $\begin{array}{l}{ }^{13} \mathrm{C} \text { NMR } \\
\text { (synthetic) } \\
\delta_{\mathrm{C}}\end{array}$ \\
\hline & & 168.4 & 168.4 \\
\hline $6.86(\mathrm{dd}, 15.8,6.0)$ & $6.87(\mathrm{dd}, 15.8,6.1)$ & 149.3 & 149.3 \\
\hline $6.07(\mathrm{dd}, 15.8,1.6)$ & $6.08(\mathrm{dd}, 15.8,1.5)$ & 123.1 & 123.1 \\
\hline $\begin{array}{l}4.94 \text { (dddd, 9.8, 7.5, 5.0, } \\
2.2 \text { ) }\end{array}$ & $4.98-4.92(\mathrm{~m})$ & 77.6 & 77.6 \\
\hline $4.48(\mathrm{ddd}, 6.0,3.0,1.6)$ & $4.50-4.47(\mathrm{~m})$ & 76.0 & 76.0 \\
\hline $3.84(\mathrm{ddd}, 6.0,4.7,3.0)$ & $3.87-3.84(\mathrm{~m})$ & 72.9 & 72.9 \\
\hline $3.98(\mathrm{q}, 6.5)$ & 3.99 (quin, 6.3) & 69.5 & 69.5 \\
\hline $\begin{array}{l}1.82(\mathrm{ddd}, 14.7,6.5,6.0) \\
1.65(\mathrm{~m})\end{array}$ & $\begin{array}{l}1.84(\mathrm{dt}, 14.7,6.1) \\
1.74-1.50(\mathrm{~m})\end{array}$ & 38.3 & 38.3 \\
\hline $1.36(\mathrm{~m})$ & $1.45-1.25(\mathrm{~m})$ & 36.8 & 36.8 \\
\hline $1.57(\mathrm{~m}), 1.61(\mathrm{~m})$ & $1.74-1.50(\mathrm{~m})$ & 36.5 & 36.5 \\
\hline $1.54(\mathrm{~m}), 1.70(\mathrm{~m})$ & $1.74-1.50(\mathrm{~m})$ & 34.1 & 34.1 \\
\hline $1.30(\mathrm{~m})$ & $1.45-1.25(\mathrm{~m})$ & 32.9 & 33.0 \\
\hline $1.15(\mathrm{~m}), 1.40(\mathrm{~m})$ & 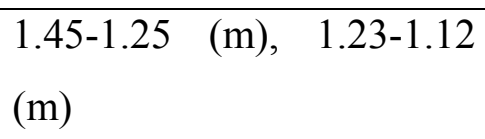 & 29.5 & 29.5 \\
\hline $1.19(\mathrm{~m}), 1.45(\mathrm{~m})$ & 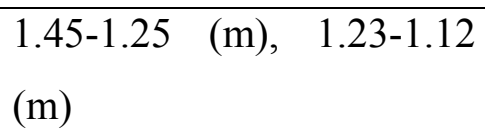 & 27.0 & 27.0 \\
\hline $1.32(\mathrm{~m})$ & $1.23-1.12(\mathrm{~m})$ & 26.4 & 26.4 \\
\hline $1.19(\mathrm{~m}), 1.37(\mathrm{~m})$ & $1.23-1.12(\mathrm{~m})$ & 25.8 & 25.8 \\
\hline $1.31(\mathrm{~m})$ & $1.23-1.12(\mathrm{~m})$ & 23.8 & 23.8 \\
\hline $0.89(\mathrm{t}, 6.8)$ & $0.91(\mathrm{t}, 6.8)$ & 14.5 & 14.5 \\
\hline
\end{tabular}


$\underline{\text { NMR comparisions of Gliomasolide C: Natural (500 MHz, CD }} \underline{3}_{2}$ OD), synthetic (400 MHz, $\underline{C D}_{3} \underline{O D}$ ):

\begin{tabular}{|c|c|c|c|}
\hline $\begin{array}{l}{ }^{1} \mathrm{H} \text { NMR (Natural) } \\
\delta_{\mathrm{H}}(\mathrm{mult}, J \text { in } \mathrm{Hz})\end{array}$ & $\begin{array}{l}{ }^{1} \mathrm{H} \text { NMR (synthetic) } \\
\delta_{\mathrm{H}}(\text { mult, } J \text { in } \mathrm{Hz} \text { ) }\end{array}$ & $\begin{array}{l}{ }^{13} \mathrm{C} \text { NMR } \\
\text { (natural) } \\
\delta_{\mathrm{C}}\end{array}$ & $\begin{array}{l}{ }^{13} \mathrm{C} \text { NMR } \\
\text { (synthetic) } \\
\delta_{\mathrm{C}}\end{array}$ \\
\hline & & 168.4 & 168.4 \\
\hline $6.18(\mathrm{dd}, 16.0,1.5)$ & $6.18(\mathrm{dd}, 15.6,1.9)$ & 123.2 & 123.2 \\
\hline $6.94(\mathrm{dd}, 16.0,5.0)$ & $6.94(\mathrm{dd}, 15.6,4.6)$ & 147.5 & 147.5 \\
\hline 4.59 (br. s) & $4.60-4.58(\mathrm{~m})$ & 73.6 & 73.6 \\
\hline $3.99(\mathrm{dd}, 3.5,3.0)$ & $3.99(\mathrm{t}, 3.4)$ & 77.3 & 77.3 \\
\hline 3.37 (br. s) & $3.37-3.36(\mathrm{~m})$ & 71.1 & 71.2 \\
\hline $4.10(\mathrm{ddd}, 7.5,5.5,1.5)$ & 4.09 (ddd, $7.8,5.4,1.7)$ & 71.2 & 71.2 \\
\hline $1.33(\mathrm{~m}), 1.68(\mathrm{~m})$ & $\begin{array}{l}1.39-1.29(\mathrm{~m}), 1.70- \\
1.62(\mathrm{~m})\end{array}$ & 32.8 & 32.8 \\
\hline $1.10(\mathrm{~m}), 1.49(\mathrm{~m})$ & $\begin{array}{l}1.17-1.07(\mathrm{~m}), 1.52-1.42 \\
(\mathrm{~m})\end{array}$ & 27.7 & 27.6 \\
\hline $1.13(\mathrm{~m}), 1.49(\mathrm{~m})$ & $\begin{array}{l}1.17-1.07(\mathrm{~m}), 1.52-1.42 \\
(\mathrm{~m})\end{array}$ & 30.3 & 30.3 \\
\hline $1.13(\mathrm{~m}), 1.34(\mathrm{~m})$ & $\begin{array}{l}1.17-1.07(\mathrm{~m}), 1.39-1.29 \\
(\mathrm{~m})\end{array}$ & 26.3 & 26.3 \\
\hline $1.46(\mathrm{~m}), 1.77(\mathrm{~m})$ & $\begin{array}{l}1.52-1.42(\mathrm{~m}), 1.77-1.71 \\
(\mathrm{~m})\end{array}$ & 34.6 & 34.5 \\
\hline $5.02(\mathrm{~m})$ & $5.03(\mathrm{~m})$ & 77.8 & 77.9 \\
\hline $1.55(\mathrm{~m}), 1.61(\mathrm{~m})$ & $1.61-1.54(\mathrm{~m})$ & 36.2 & 36.2 \\
\hline $1.34(\mathrm{~m})$ & $1.39-1.29(\mathrm{~m})$ & 26.3 & 26.3 \\
\hline $1.33(\mathrm{~m})$ & $1.39-1.29(\mathrm{~m})$ & 32.8 & 32.8 \\
\hline $1.33(\mathrm{~m})$ & $1.39-1.29(\mathrm{~m})$ & 23.6 & 23.6 \\
\hline $0.92(\mathrm{t}, 7.0)$ & $0.91(t, 6.6)$ & 14.4 & 14.3 \\
\hline
\end{tabular}




\section{Attempts to prepare Sch-725674 from compound 21:}

We have attempted to synthesize compound $\mathbf{1}$ from $\mathbf{2 1}$ through Wacker oxidation of $\mathbf{2 1}$ to give compound 13 (known intermediate from Kaliappan's synthesis) which was previously transformed to Sch-725674 (reference 8). However, all our attempts using Wacker oxidation conditions were not successful to obtain desired compound 13. All the conditions we have attempted are summarized below.

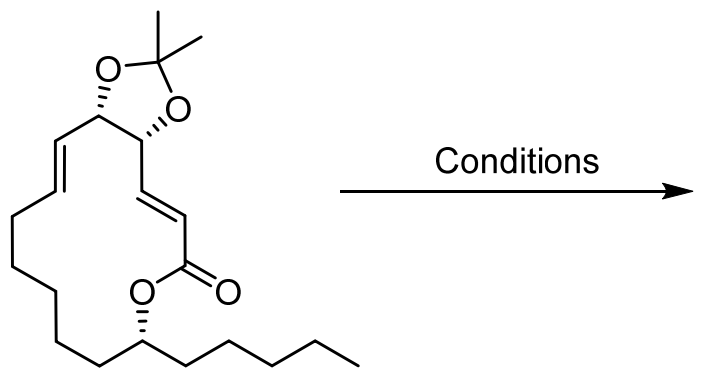

21

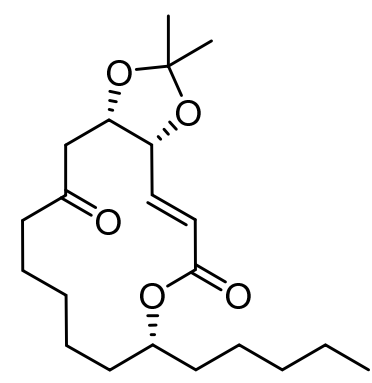

13

\begin{tabular}{|c|c|c|c|c|c|}
\hline $\begin{array}{c}\text { S.N } \\
\text { o }\end{array}$ & Reagent & Solvent & $\begin{array}{l}\text { Temp., } \\
{ }^{\circ} \mathrm{C}\end{array}$ & $\begin{array}{l}\text { Reaction } \\
\text { time }\end{array}$ & Result \\
\hline 1 & $\begin{array}{l}\mathrm{PdCl}_{2}, \mathrm{CuCl}, \\
\mathrm{O}_{2} \text { (balloon) }\end{array}$ & $\begin{array}{l}\text { DMA- } \mathrm{H}_{2} \mathrm{O} \\
(4: 1)\end{array}$ & $80{ }^{\circ} \mathrm{C}$ & $48 \mathrm{~h}$ & $\begin{array}{c}\text { Only starting material } \\
\text { recovered }\end{array}$ \\
\hline 2 & $\begin{array}{c}\mathrm{Pd}(\mathrm{OAc})_{2}, \\
\mathrm{HBF} 4, \\
\text { benzoquinone }\end{array}$ & DMA:ACN: $\mathrm{H}_{2} \mathrm{O}$ & $\mathrm{RT}-60{ }^{\circ} \mathrm{C}$ & $24 \mathrm{~h}$ & $\begin{array}{l}\text { Only starting material } \\
\text { recovered }\end{array}$ \\
\hline 3 & $\begin{array}{c}\mathrm{Pd}\left(\mathrm{CH}_{3} \mathrm{CN}\right)_{2} \mathrm{Cl}_{2} \\
\text { AgSbF6, } \\
\text { aq.TBHP }\end{array}$ & $\mathrm{CH}_{2} \mathrm{Cl}_{2}$ & RT & $16 \mathrm{~h}$ & $\begin{array}{l}\text { TLC showed complex } \\
\text { reaction mixture }\end{array}$ \\
\hline 4 & $\begin{array}{l}\mathrm{PdCl}_{2}, \mathrm{O}_{2}(250 \\
\text { psi })\end{array}$ & $\begin{array}{l}\text { DMA- } \mathrm{H}_{2} \mathrm{O} \\
\quad(4: 1)\end{array}$ & $80^{\circ} \mathrm{C}$ & $48 \mathrm{~h}$ & $\begin{array}{c}\text { Only starting material } \\
\text { recovered }\end{array}$ \\
\hline 5 & $\begin{array}{l}\mathrm{PdCl}_{2}, \mathrm{O}_{2}(250 \\
\text { psi })\end{array}$ & $\begin{array}{l}\text { DMF- } \mathrm{H}_{2} \mathrm{O} \\
(4: 1)\end{array}$ & $80{ }^{\circ} \mathrm{C}$ & $24 \mathrm{~h}$ & $\begin{array}{c}\text { Only starting material } \\
\text { recovered }\end{array}$ \\
\hline 6 & $\begin{array}{l}\mathrm{PdCl}_{2}, \mathrm{O}_{2}(400 \\
\text { psi })\end{array}$ & $\begin{array}{l}\text { DMA- } \mathrm{H}_{2} \mathrm{O} \\
(4: 1)\end{array}$ & $100{ }^{\circ} \mathrm{C}$ & $48 \mathrm{~h}$ & $\begin{array}{l}\text { Only starting material } \\
\text { recovered }\end{array}$ \\
\hline
\end{tabular}




\section{Attempts to synthesize Gliomasilode $\mathrm{C}$ from compound 21:}

We have also attempted to synthesize Gliomasilode $\mathrm{C}$ natural product starting from the macrocycle 21 using selective dihydroxylation $\left(\mathrm{OsO}_{4} / \mathrm{NMO}\right.$ conditions) of isolated double bond. However, our attempts resulted in formation of undesired compounds such as 21a and 21b.

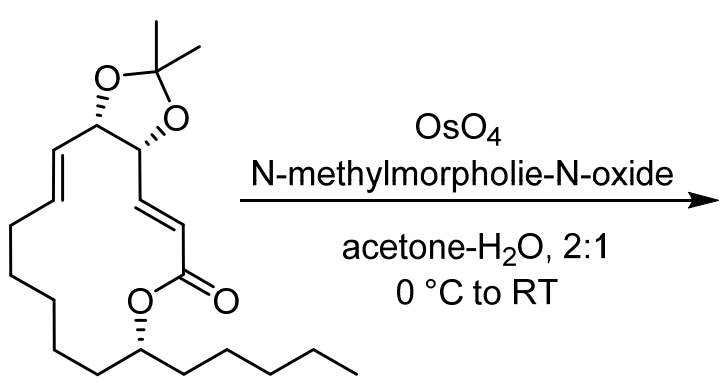

21

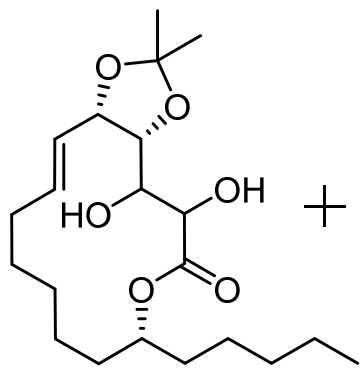

21 a (less polar spot)<smiles>CCCCC[C@@H]1CCCCCC(O)C(O)[C@H]2OC(C)(C)O[C@H]2C1C(O)C(=O)OC1OC(C)(C)O1</smiles>

21b (more polar spot)

tentative strucures 
${ }^{1} \mathrm{H}$ NMR spectrum of compound $7\left(\mathrm{CDCl}_{3}, 400 \mathrm{MHz}\right)$

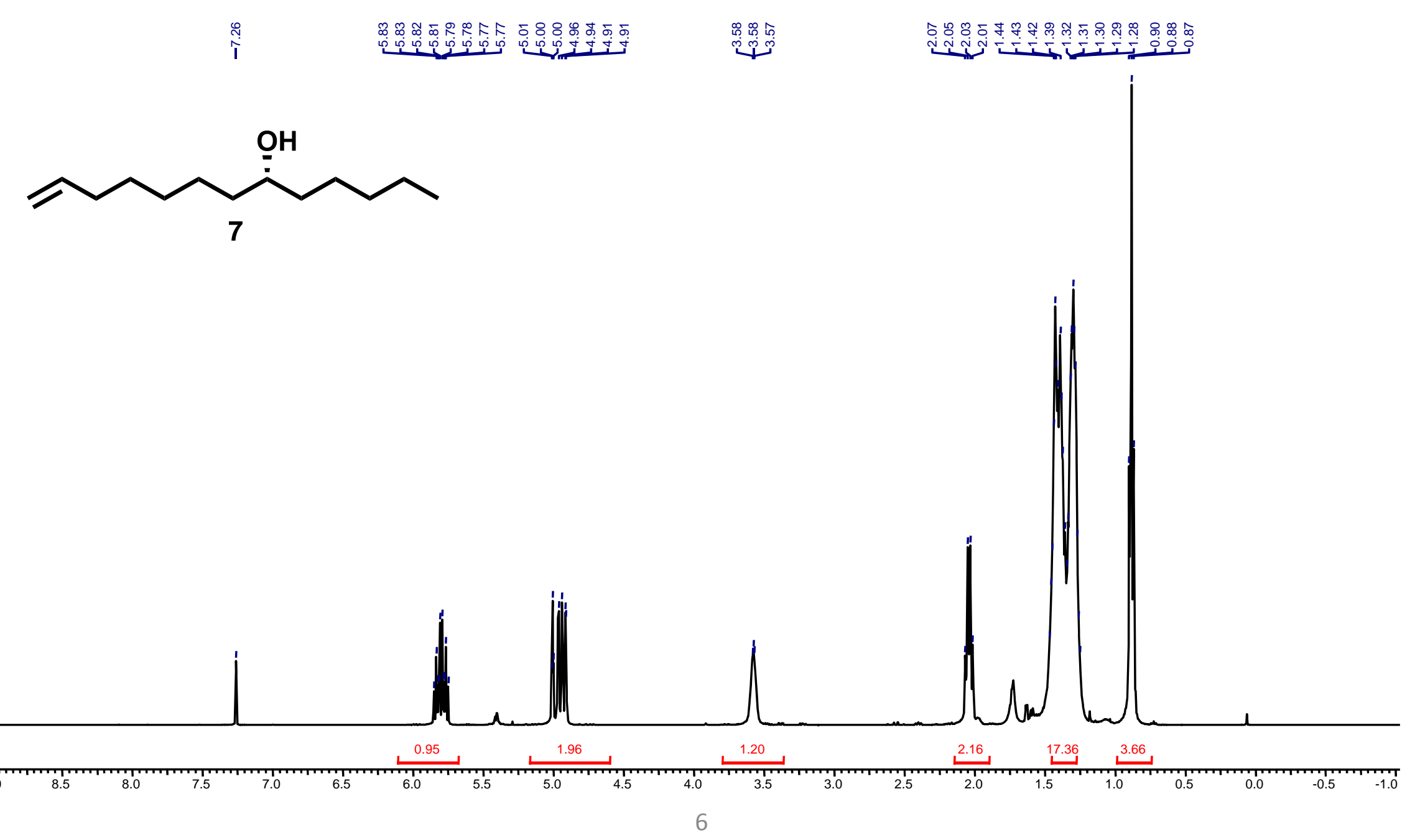


${ }^{13} \mathrm{C}$ NMR spectrum of compound $7\left(\mathrm{CDCl}_{3}, 100 \mathrm{MHz}\right)$

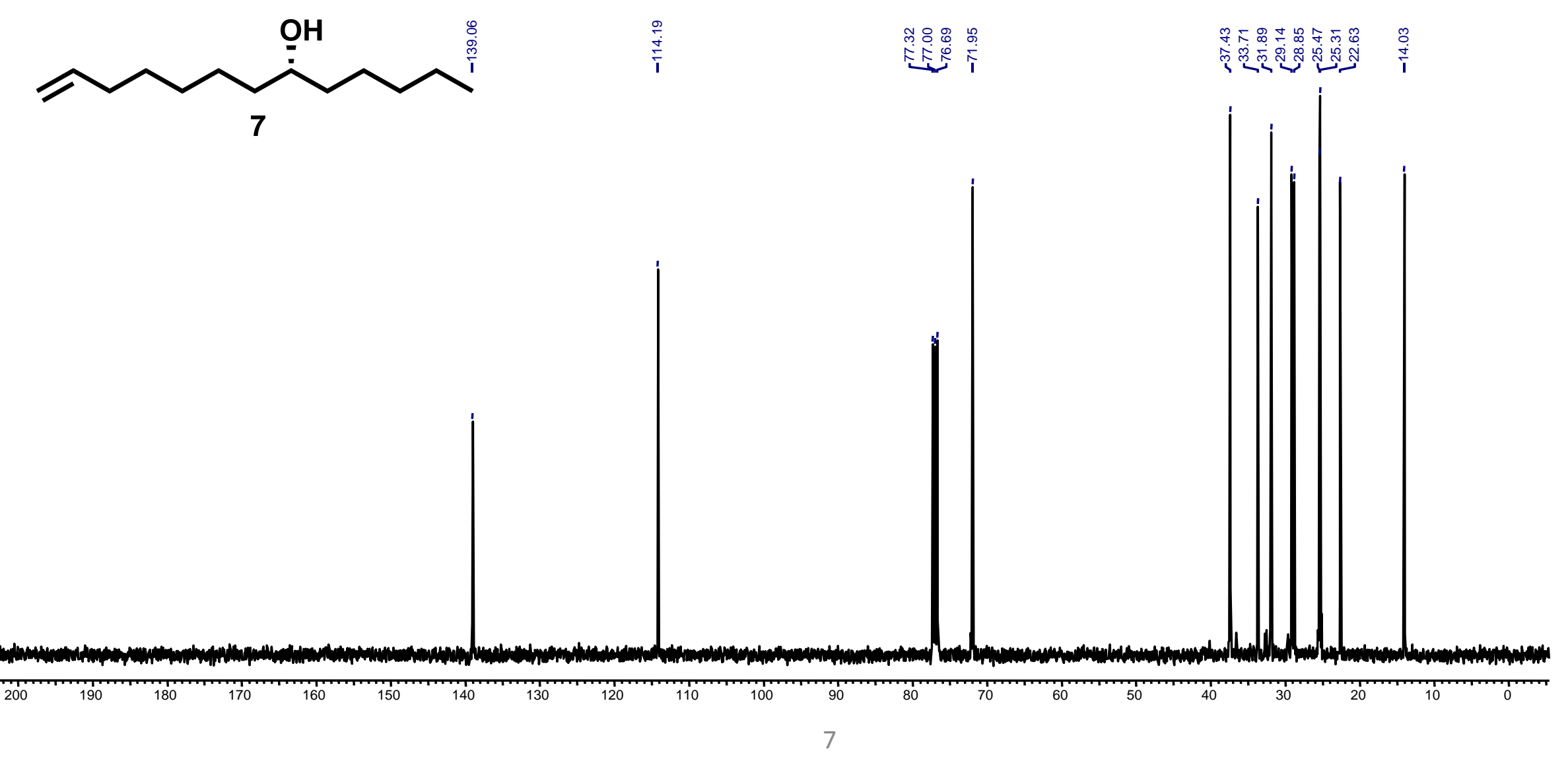


${ }^{1} \mathrm{H}$ NMR spectrum of compound $10\left(\mathrm{CDCl}_{3}, 400 \mathrm{MHz}\right)$

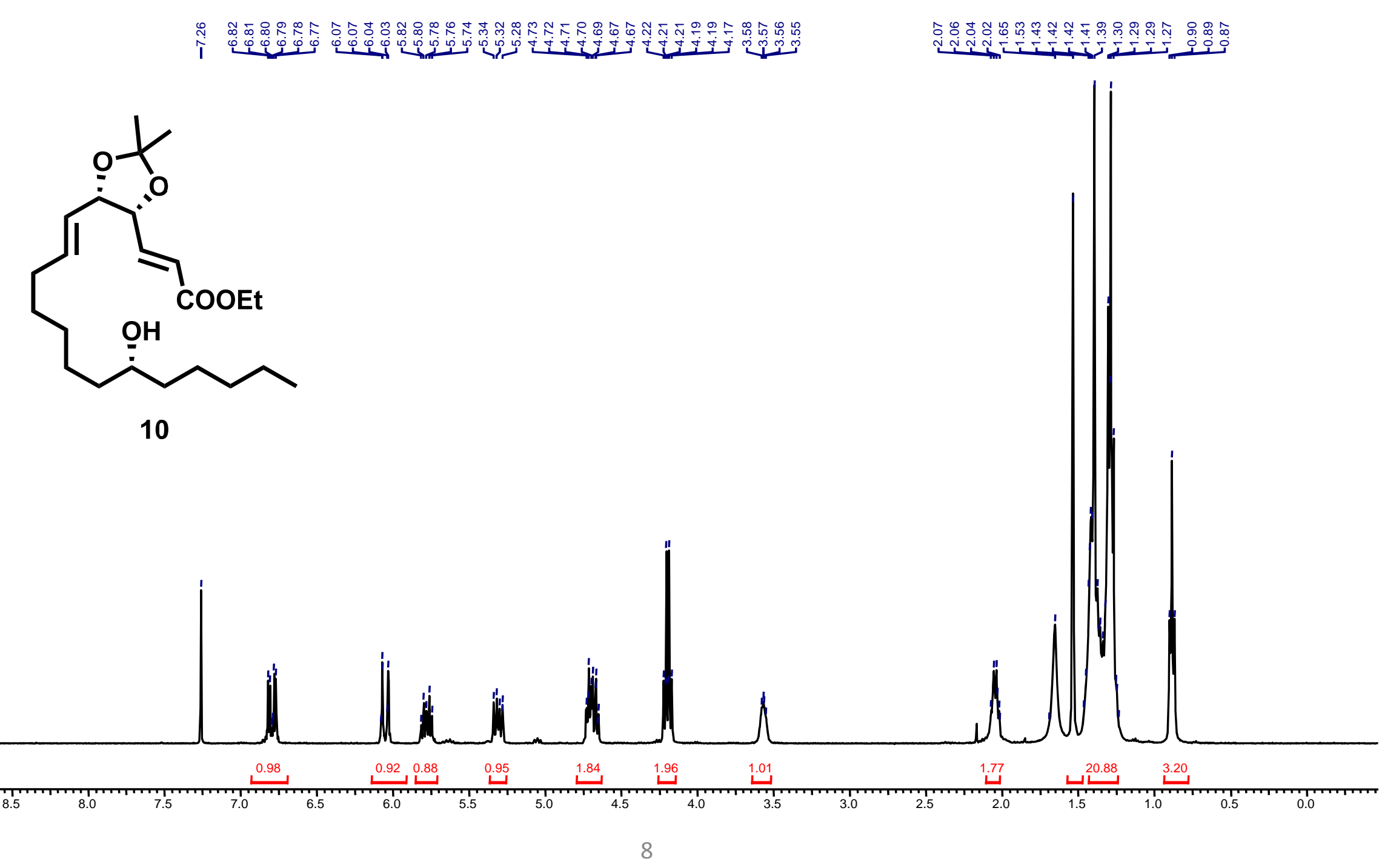


${ }^{13} \mathrm{C}$ NMR spectrum of compound $10\left(\mathrm{CDCl}_{3}, 100 \mathrm{MHz}\right)$

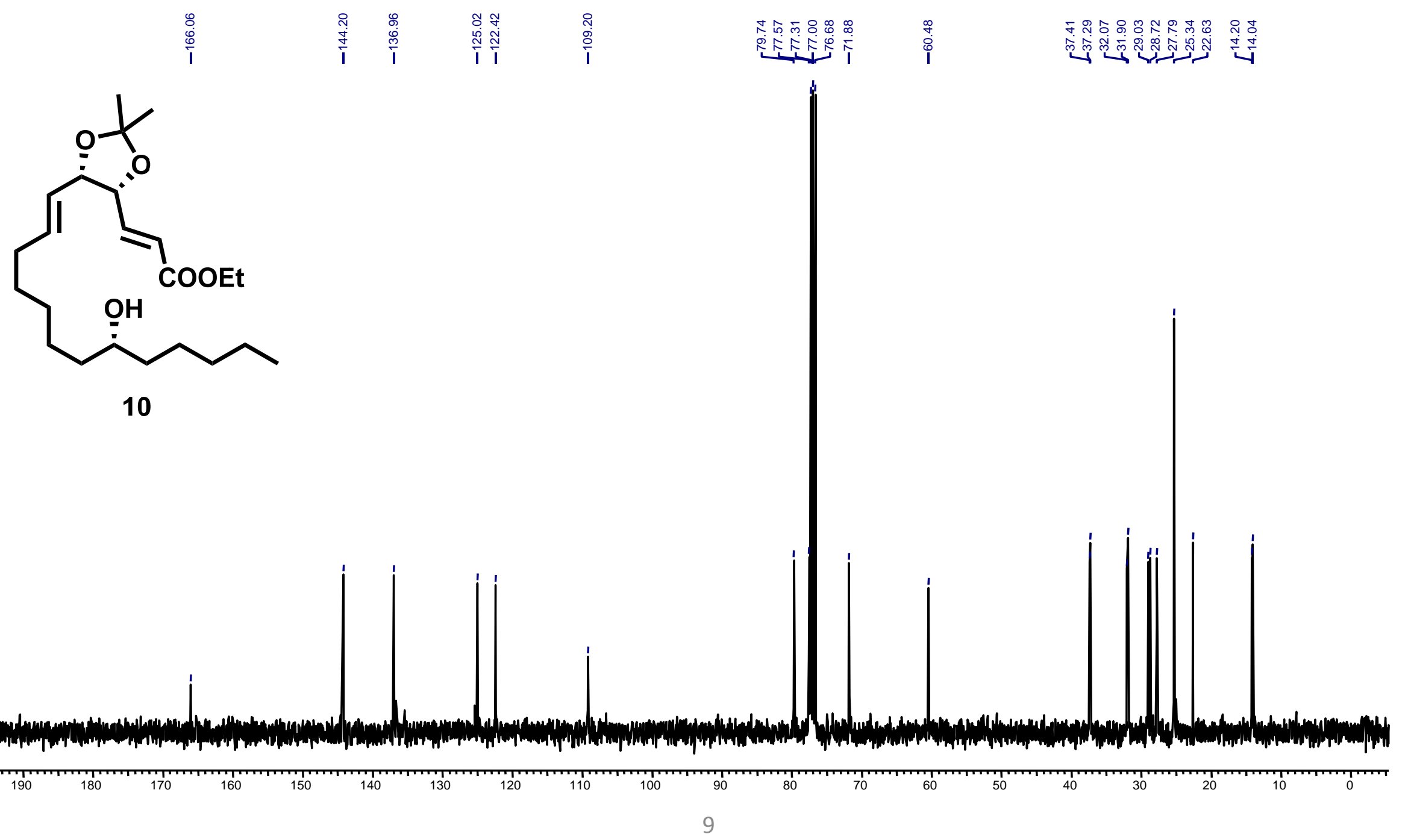


${ }^{1} \mathrm{H}$ NMR spectrum of compound $11\left(\mathrm{CDCl}_{3}, 400 \mathrm{MHz}\right)$

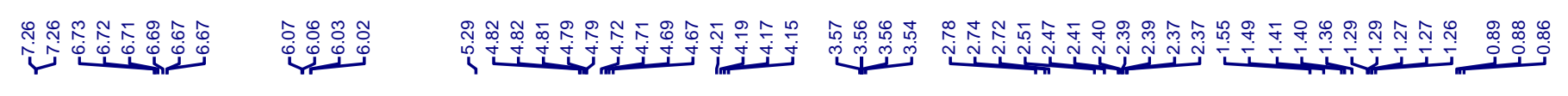

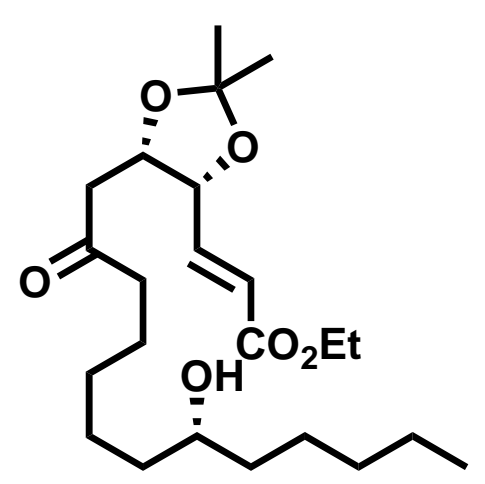

11

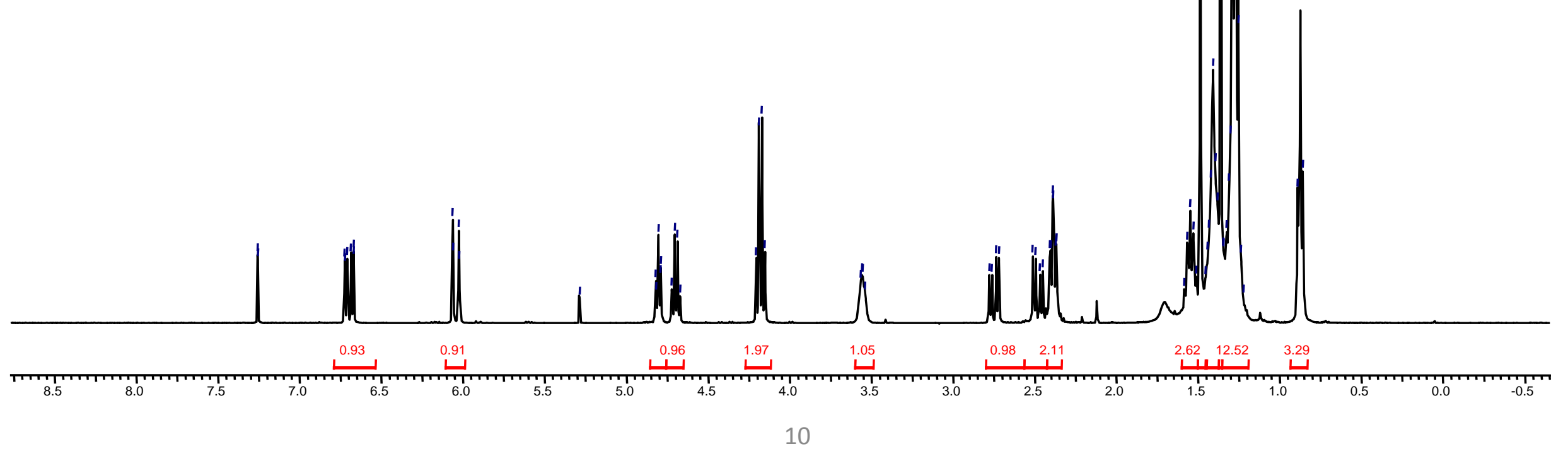


${ }^{13} \mathrm{C}$ NMR spectrum of compound $11\left(\mathrm{CDCl}_{3}, 100 \mathrm{MHz}\right)$

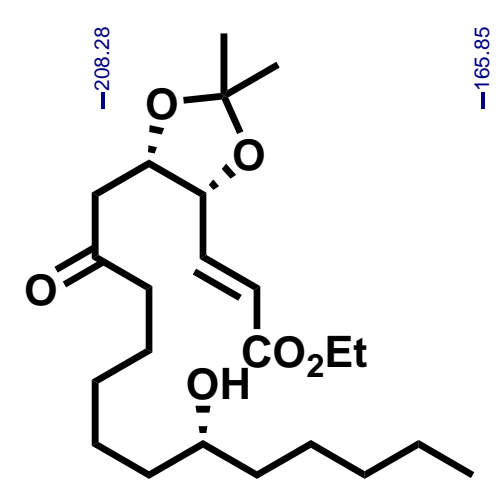

11

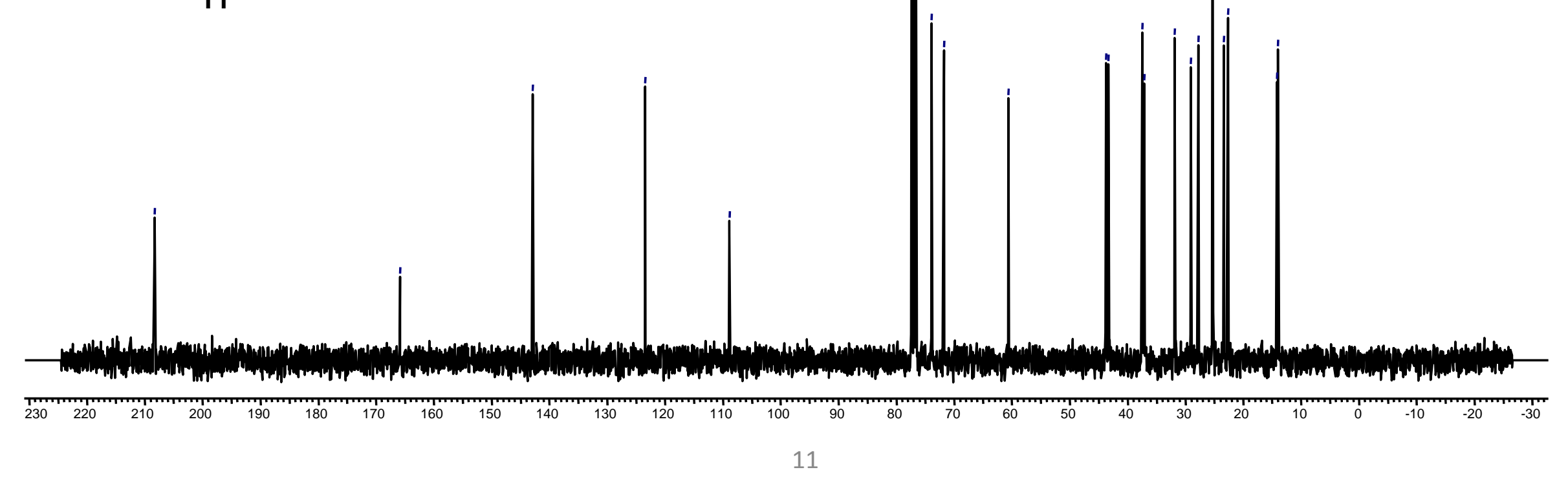


${ }^{1} \mathrm{H}$ NMR spectrum of compound $12\left(\mathrm{CDCl}_{3}, 400 \mathrm{MHz}\right)$

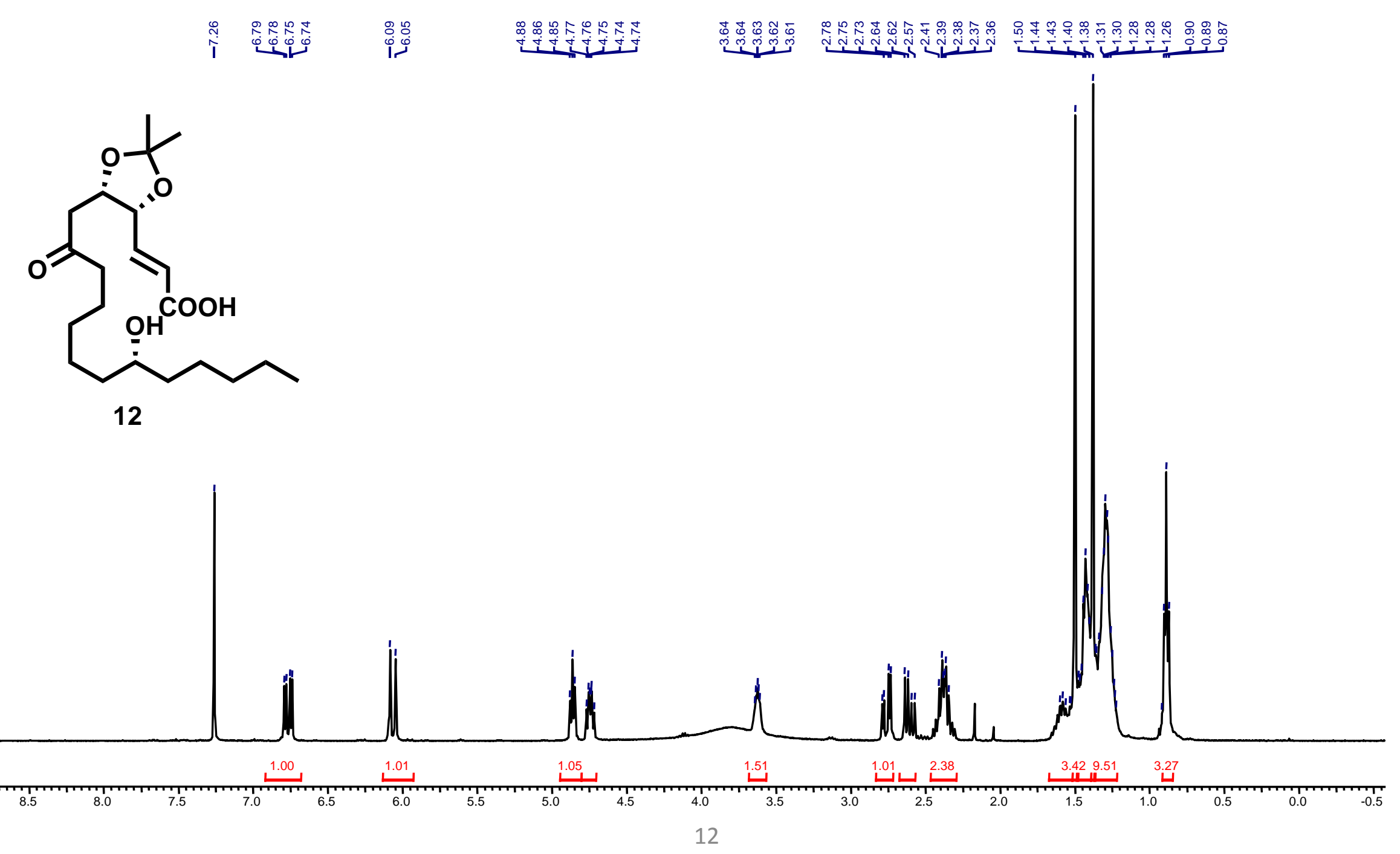


${ }^{13} \mathrm{C}$ NMR spectrum of compound $12\left(\mathrm{CDCl}_{3}, 100 \mathrm{MHz}\right)$

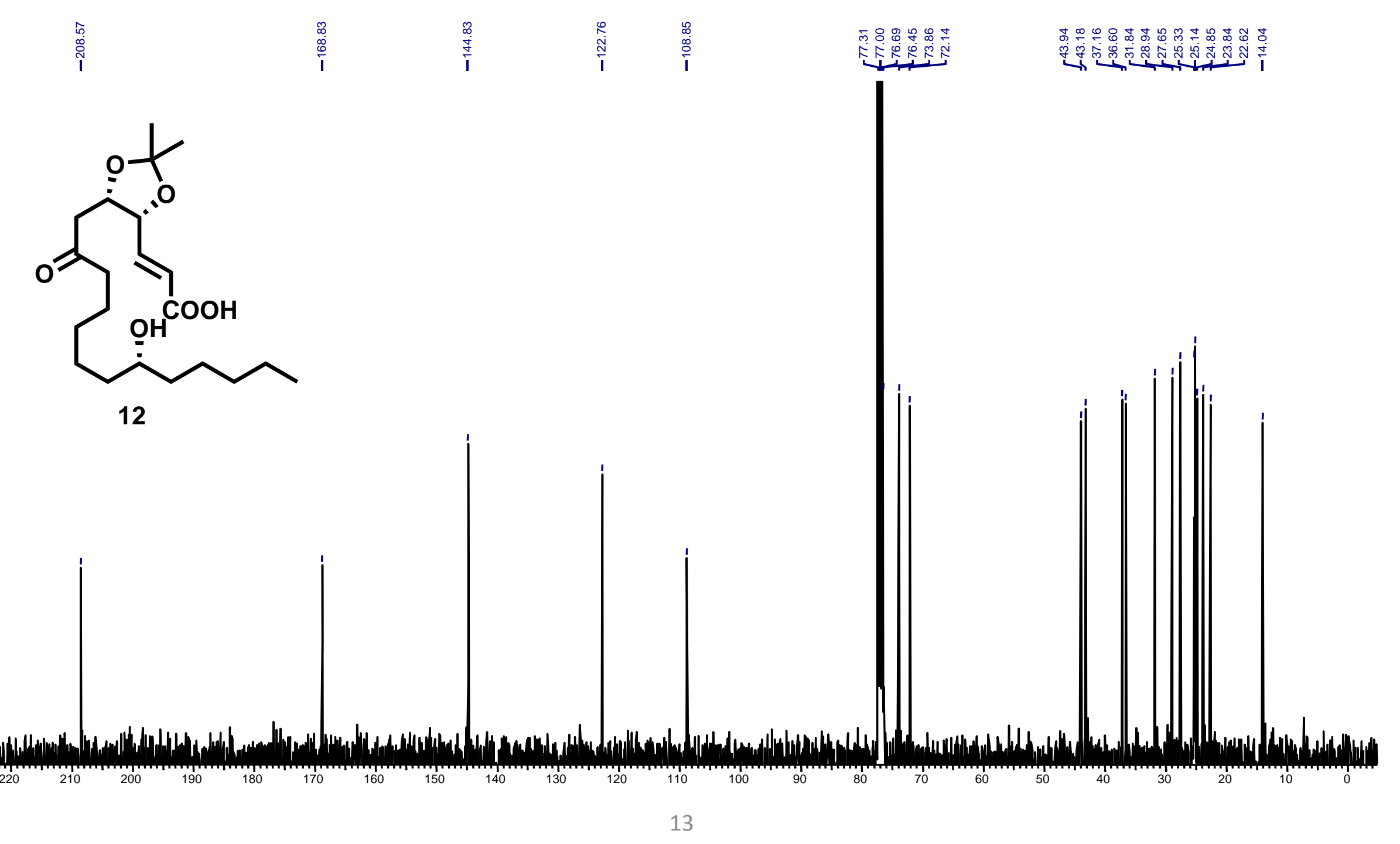




\section{${ }^{1} \mathrm{H}$ NMR spectrum of compound $13\left(\mathrm{CDCl}_{3}, 400 \mathrm{MHz}\right)$}

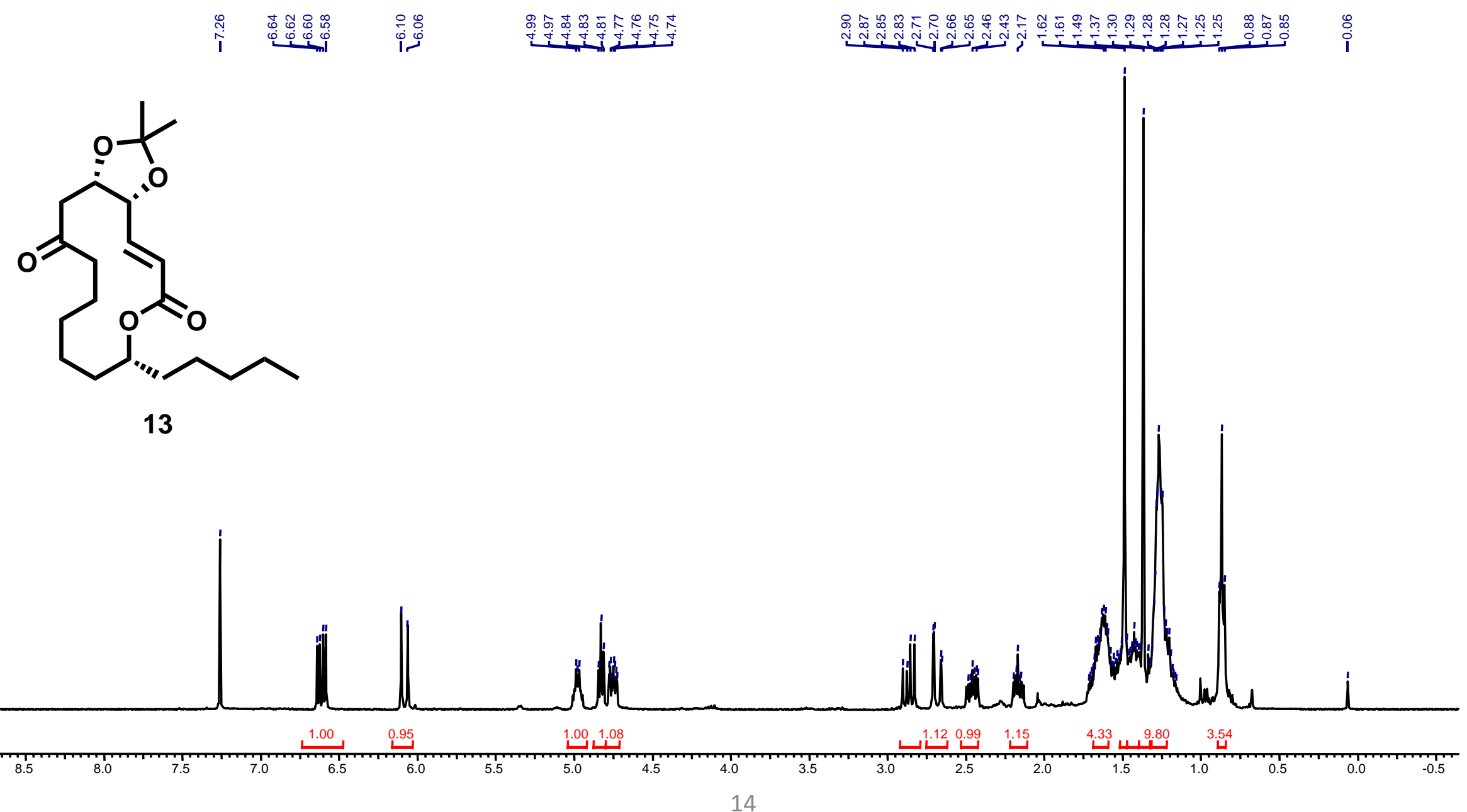


${ }^{13} \mathrm{C}$ NMR spectrum of compound $13\left(\mathrm{CDCl}_{3}, 100 \mathrm{MHz}\right)$

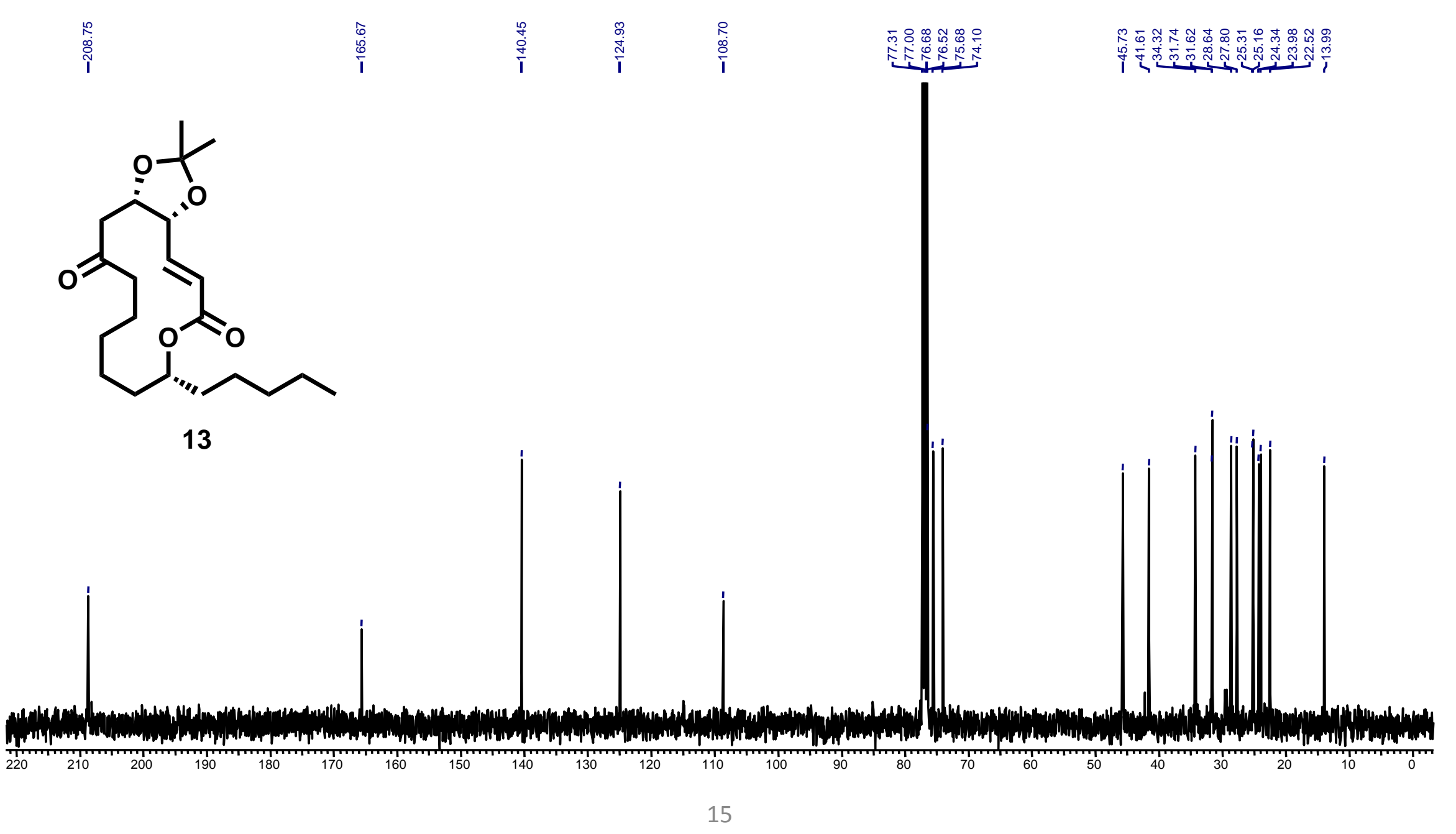




\section{${ }^{1} \mathrm{H}$ NMR spectrum of compound $14\left(\mathrm{CDCl}_{3}, 400 \mathrm{MHz}\right)$}

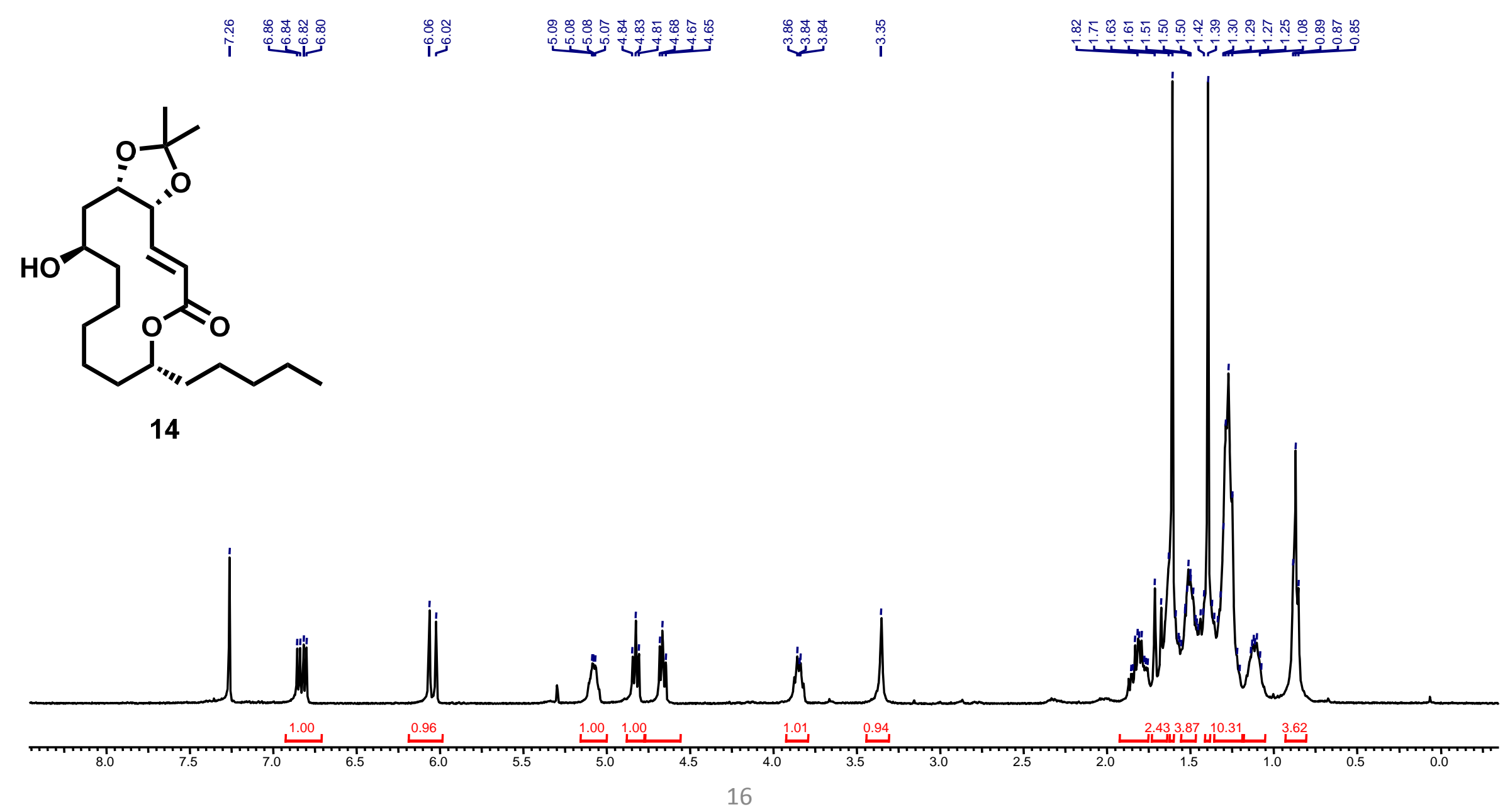


${ }^{13} \mathrm{C}$ NMR spectrum of compound $14\left(\mathrm{CDCl}_{3}, 100 \mathrm{MHz}\right)$

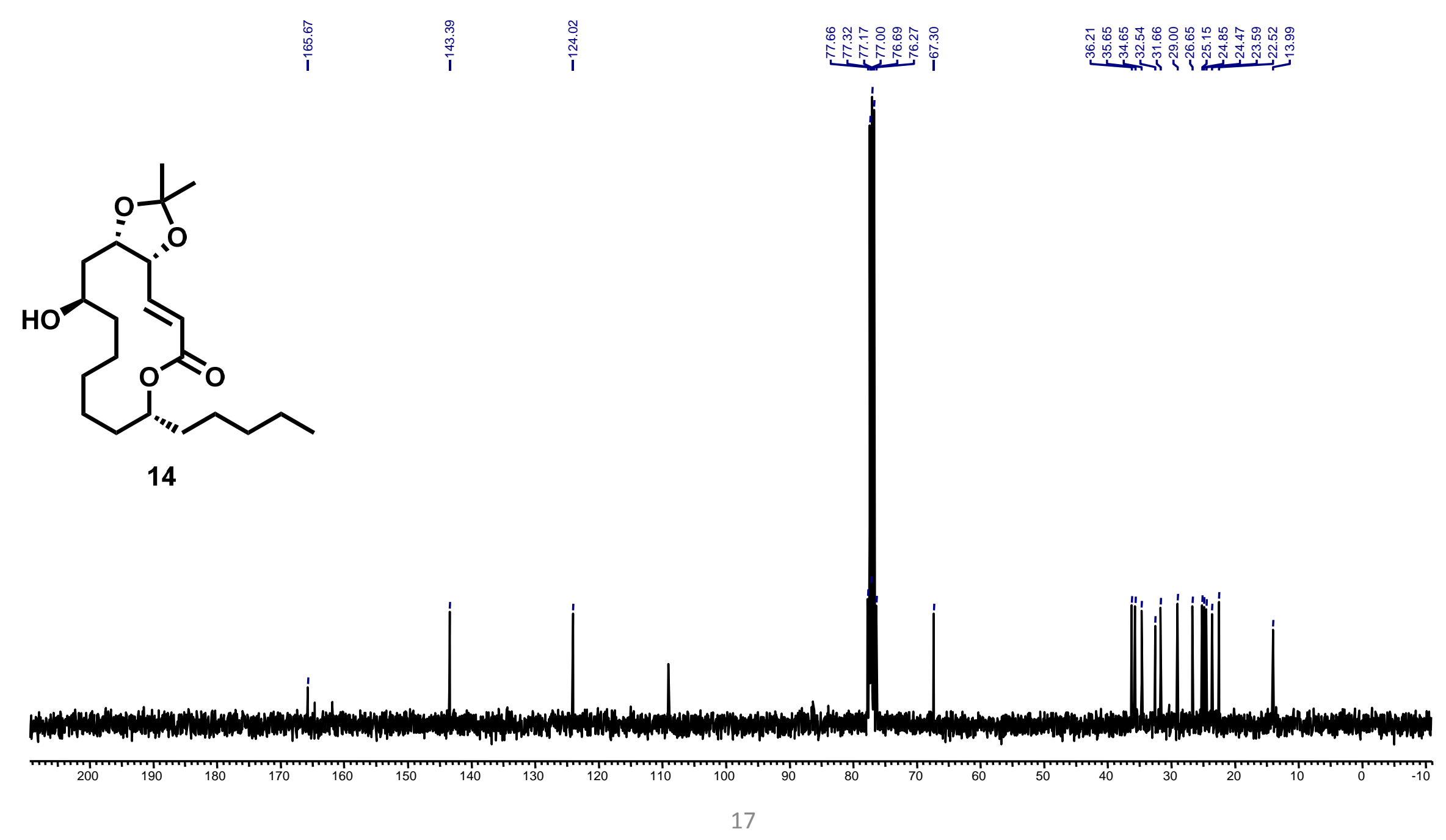




\section{${ }^{1} \mathrm{H}$ NMR spectrum of compound $1\left(\mathrm{CD}_{3} \mathrm{OD}, 400 \mathrm{MHz}\right)$}

Methanol; Layer No.: 1; Created by: Administrator; Created at: 07.23.2015 17:54:24 (GMT05:30)

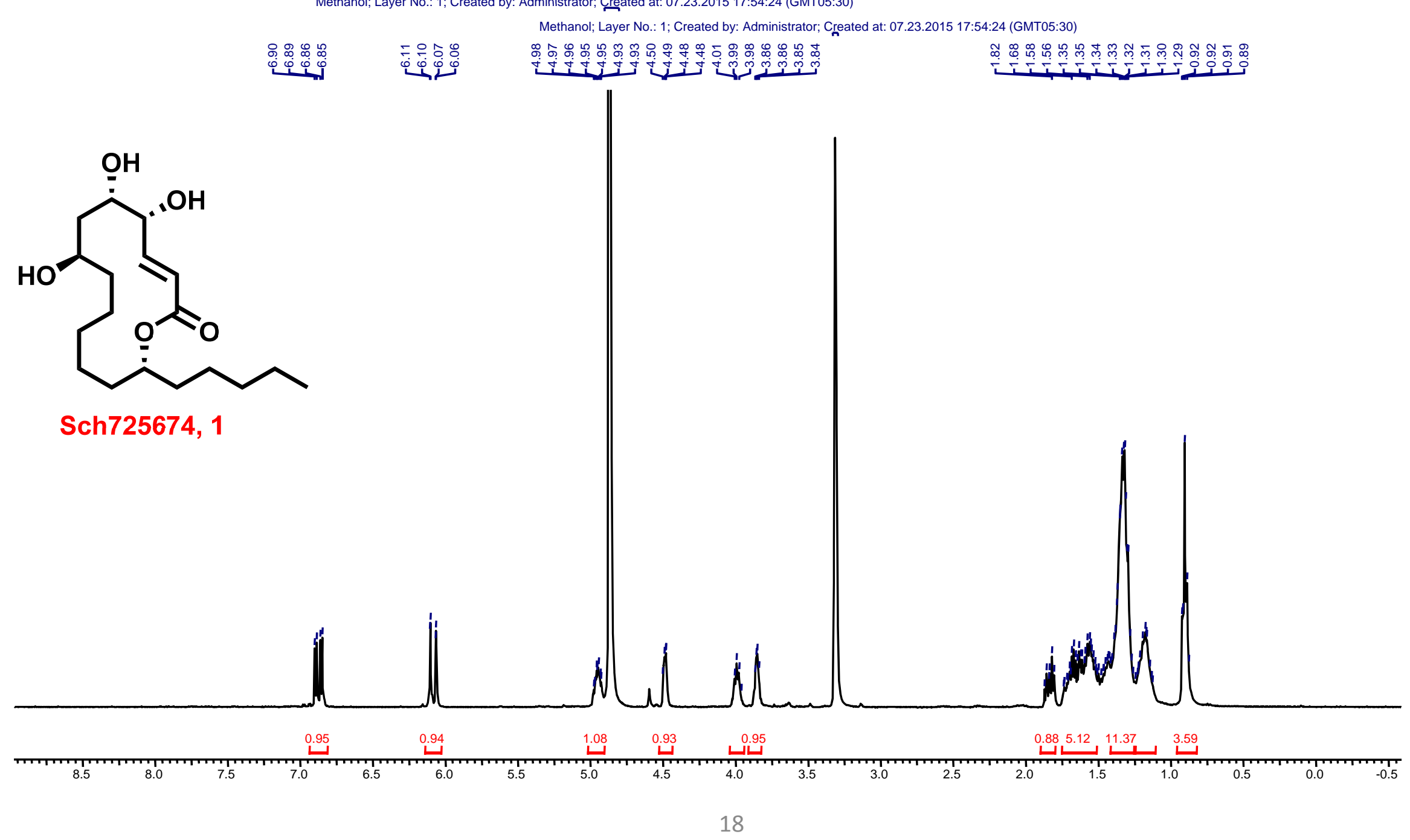




\section{${ }^{13} \mathrm{C}$ NMR spectrum of compound $1\left(\mathrm{CD}_{3} \mathrm{OD}, 100 \mathrm{MHz}\right)$}

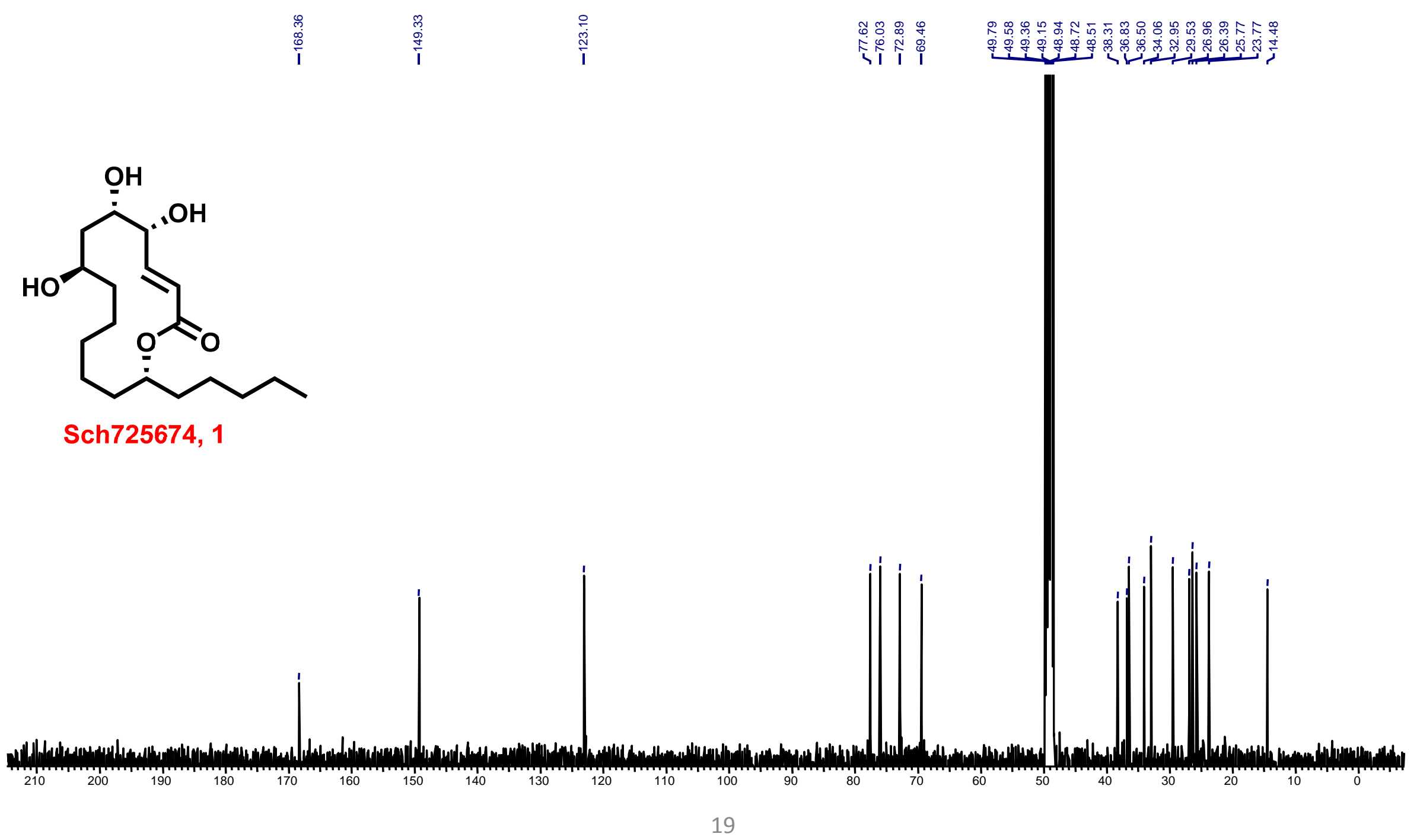


${ }^{1} \mathrm{H}$ NMR spectrum of compound $9\left(\mathrm{CDCl}_{3}, 400 \mathrm{MHz}\right)$

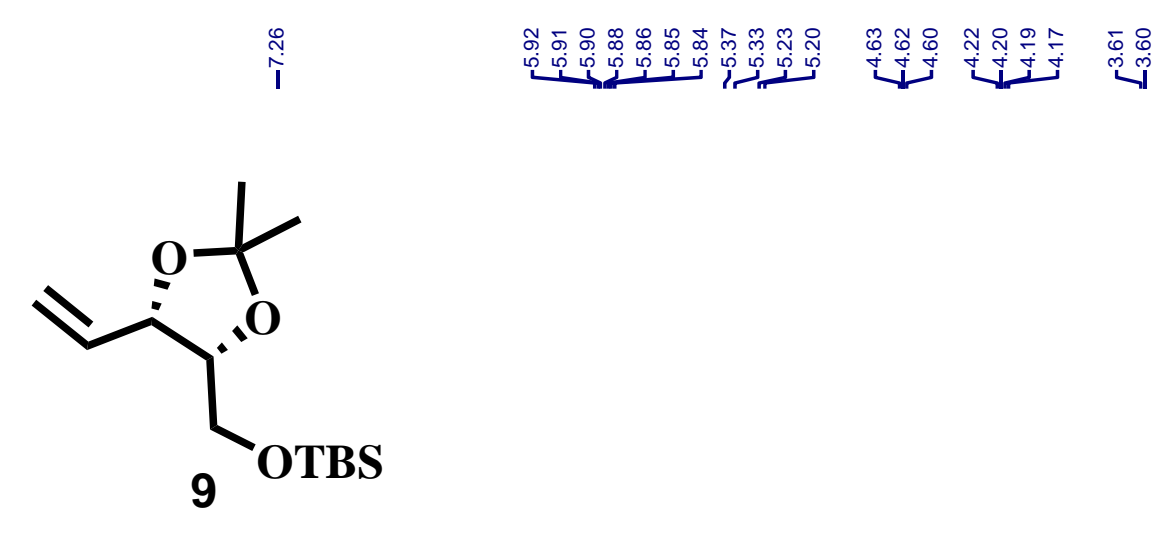


${ }^{13} \mathrm{C}$ NMR spectrum of compound $9\left(\mathrm{CDCl}_{3}, 100 \mathrm{MHz}\right)$

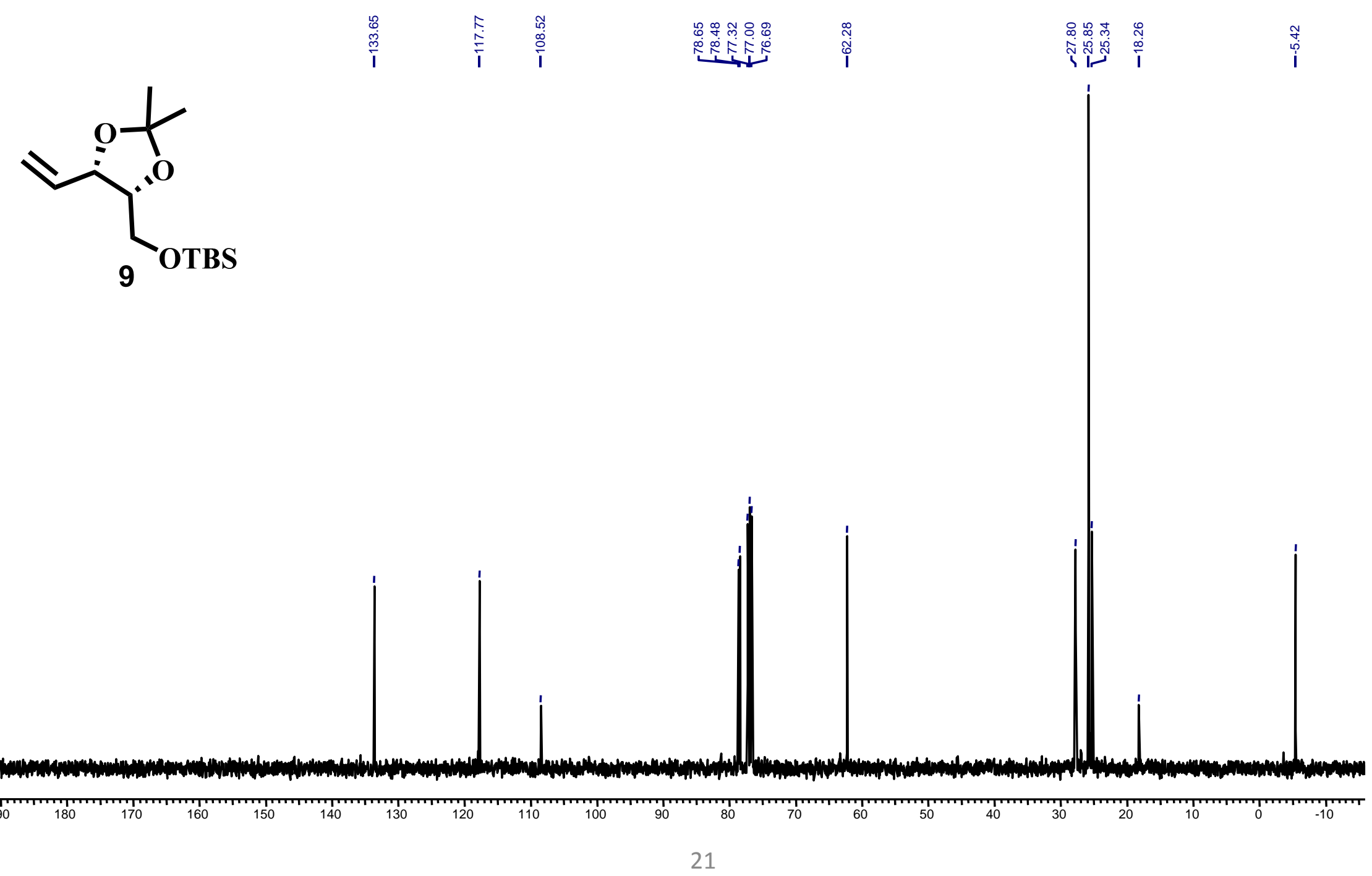




\section{${ }^{1} \mathrm{H}$ NMR spectrum of compound $15\left(\mathrm{CDCl}_{3}, 400 \mathrm{MHz}\right)$}

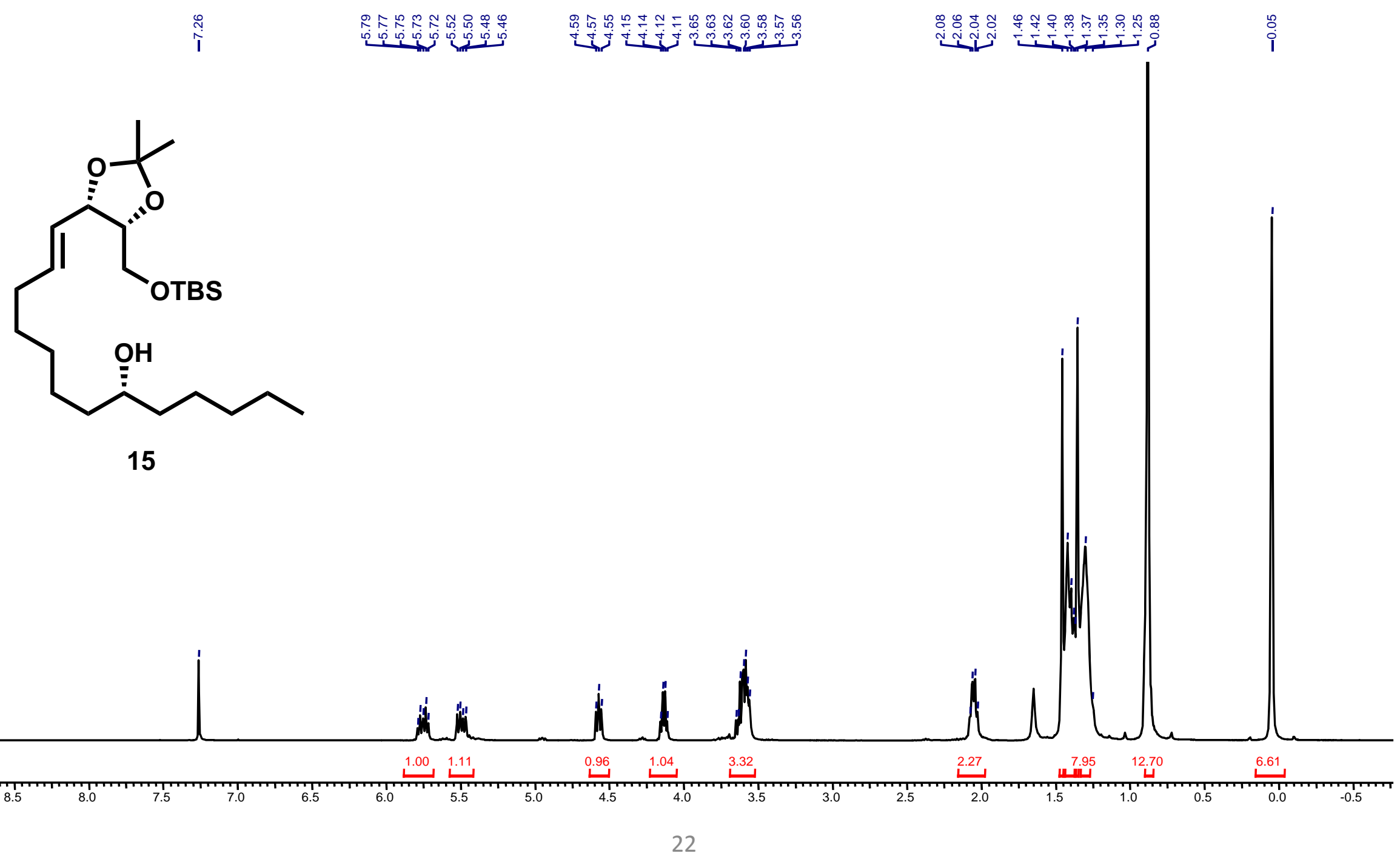


${ }^{13} \mathrm{C}$ NMR spectrum of compound $15\left(\mathrm{CDCl}_{3}, 100 \mathrm{MHz}\right)$

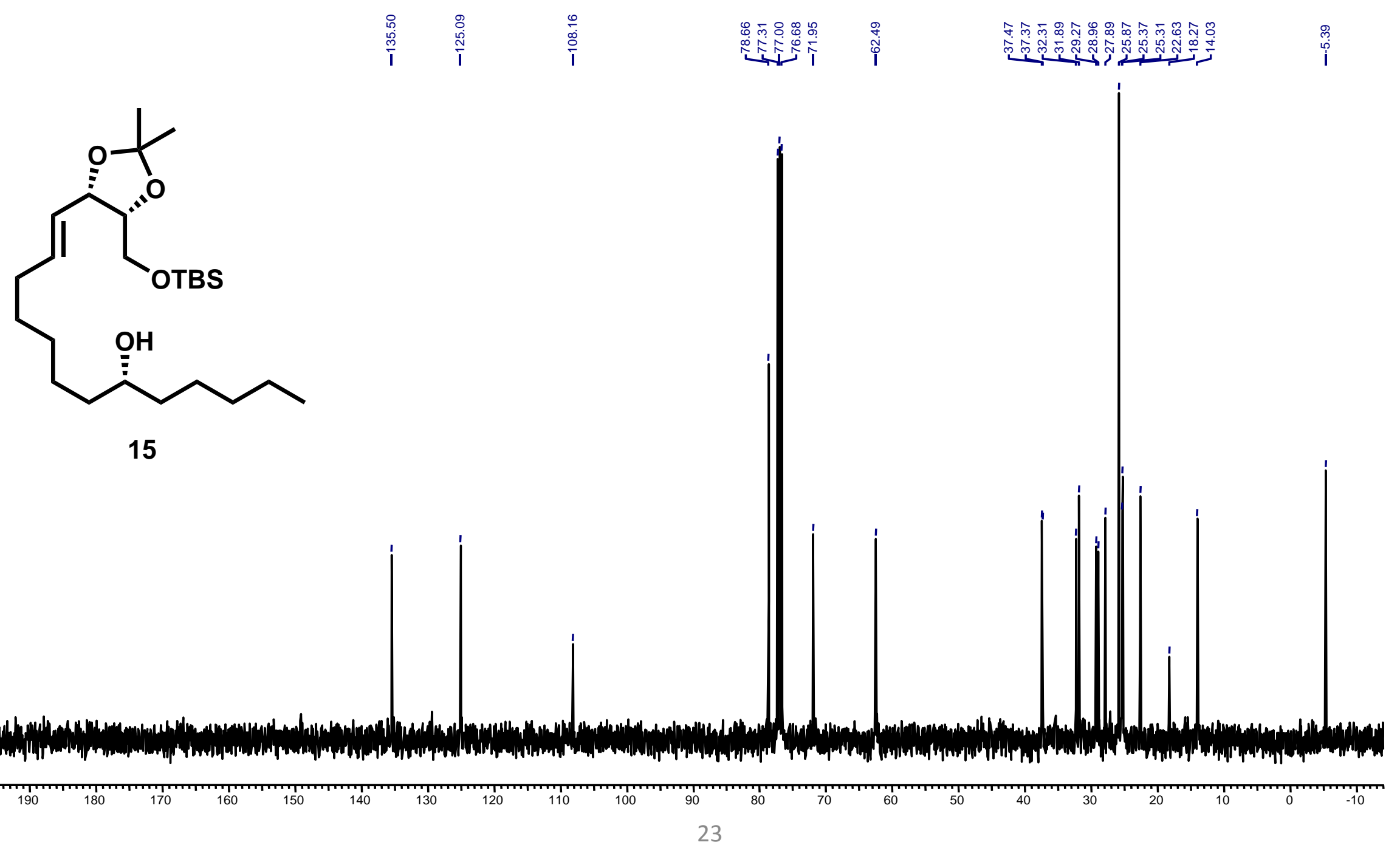




\section{${ }^{1} \mathrm{H}$ NMR spectrum of compound $16\left(\mathrm{CDCl}_{3}, 400 \mathrm{MHz}\right)$}

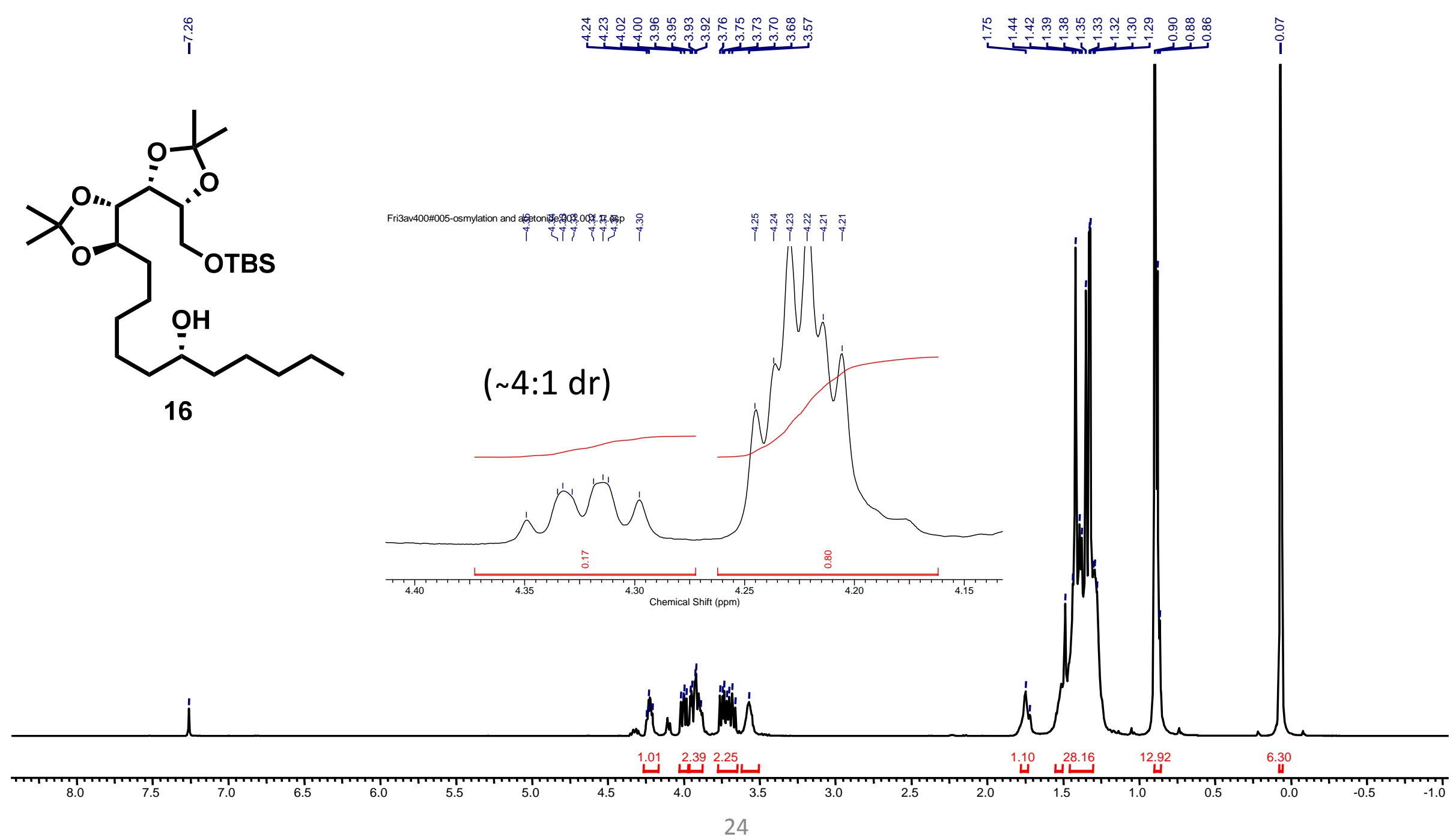


${ }^{13} \mathrm{C}$ NMR spectrum of compound $16\left(\mathrm{CDCl}_{3}, 100 \mathrm{MHz}\right)$

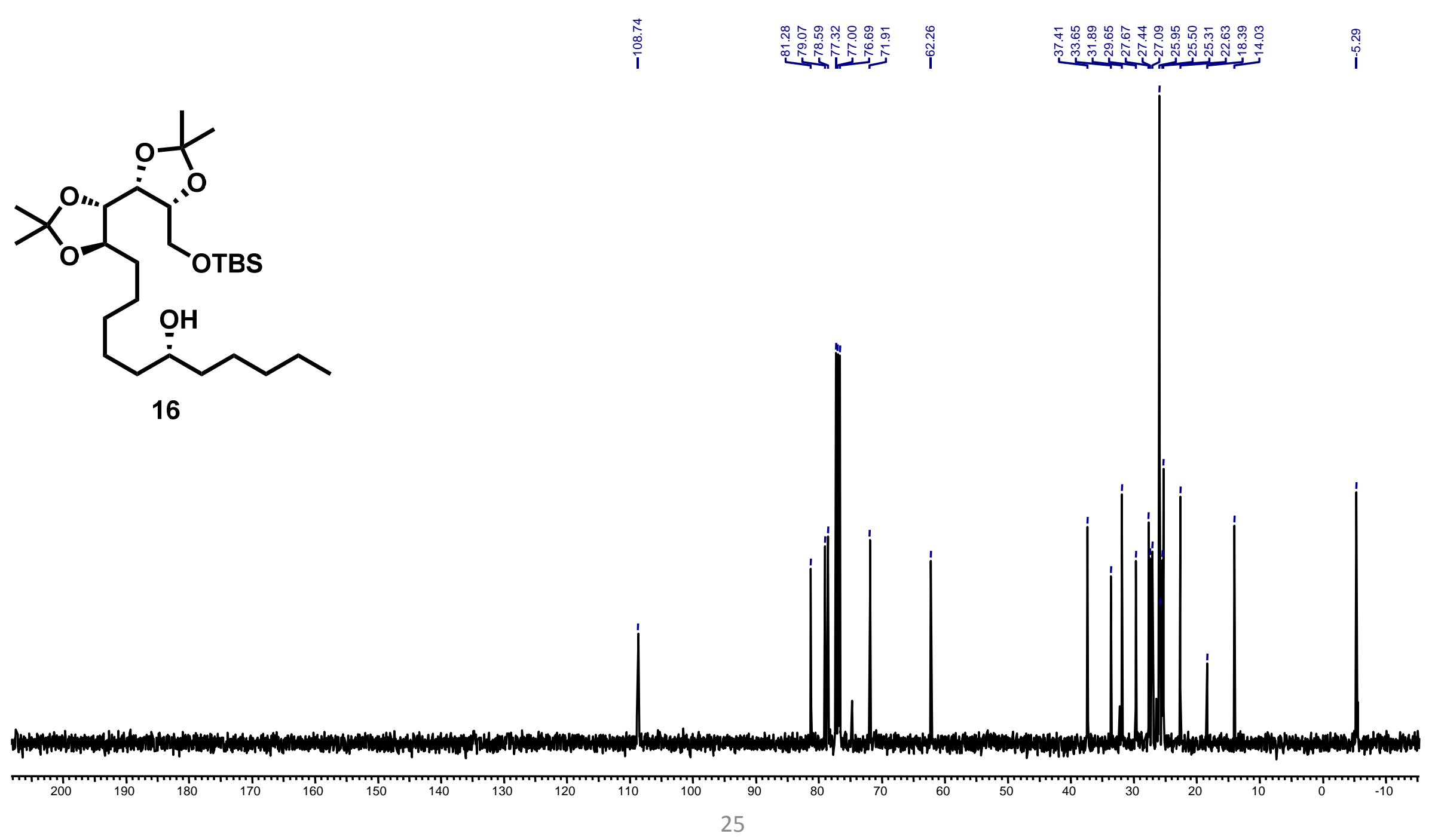




\section{${ }^{1} \mathrm{H}$ NMR spectrum of compound $17\left(\mathrm{CDCl}_{3}, 400 \mathrm{MHz}\right)$}

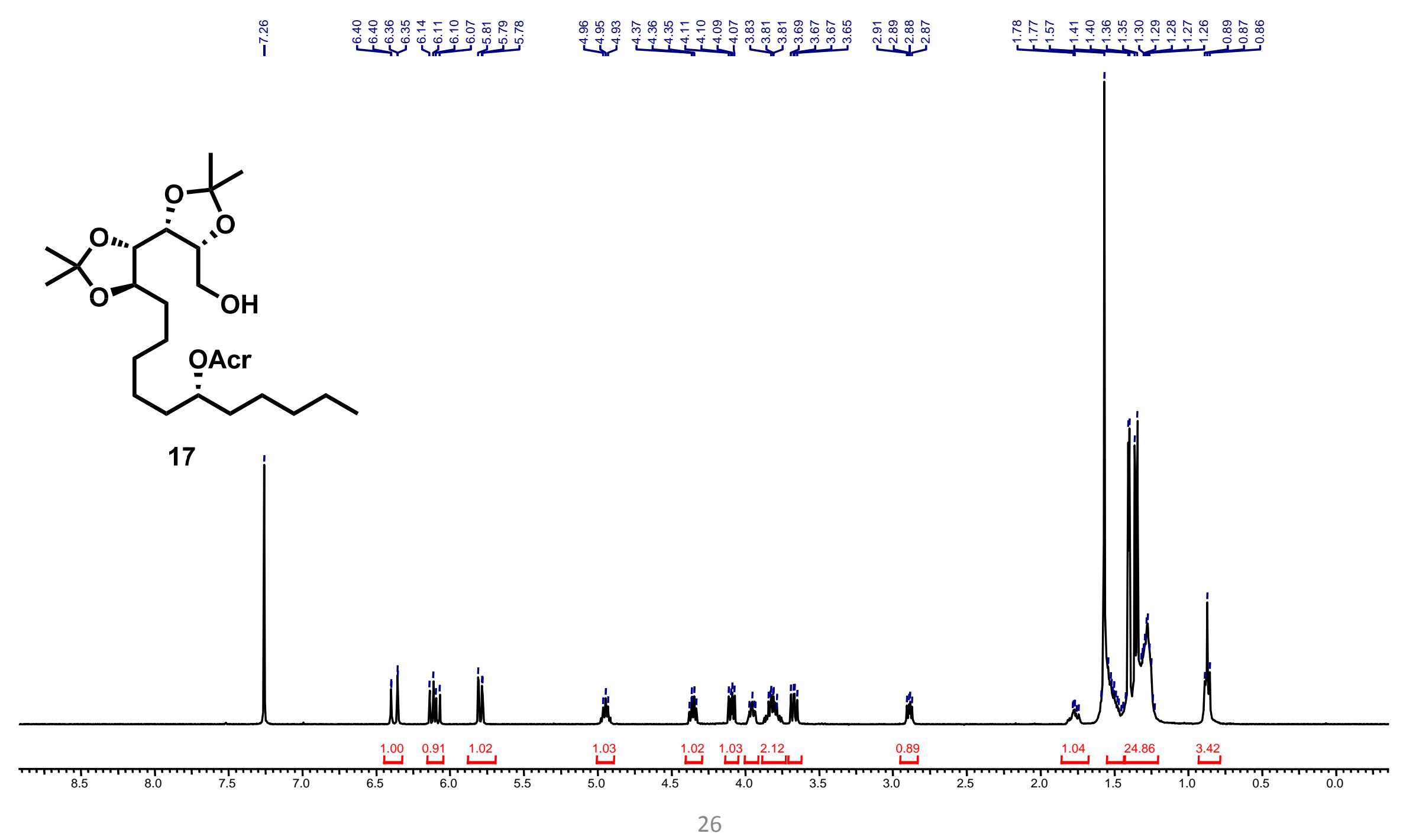


${ }^{13} \mathrm{C}$ NMR spectrum of compound $17\left(\mathrm{CDCl}_{3}, 125 \mathrm{MHz}\right)$

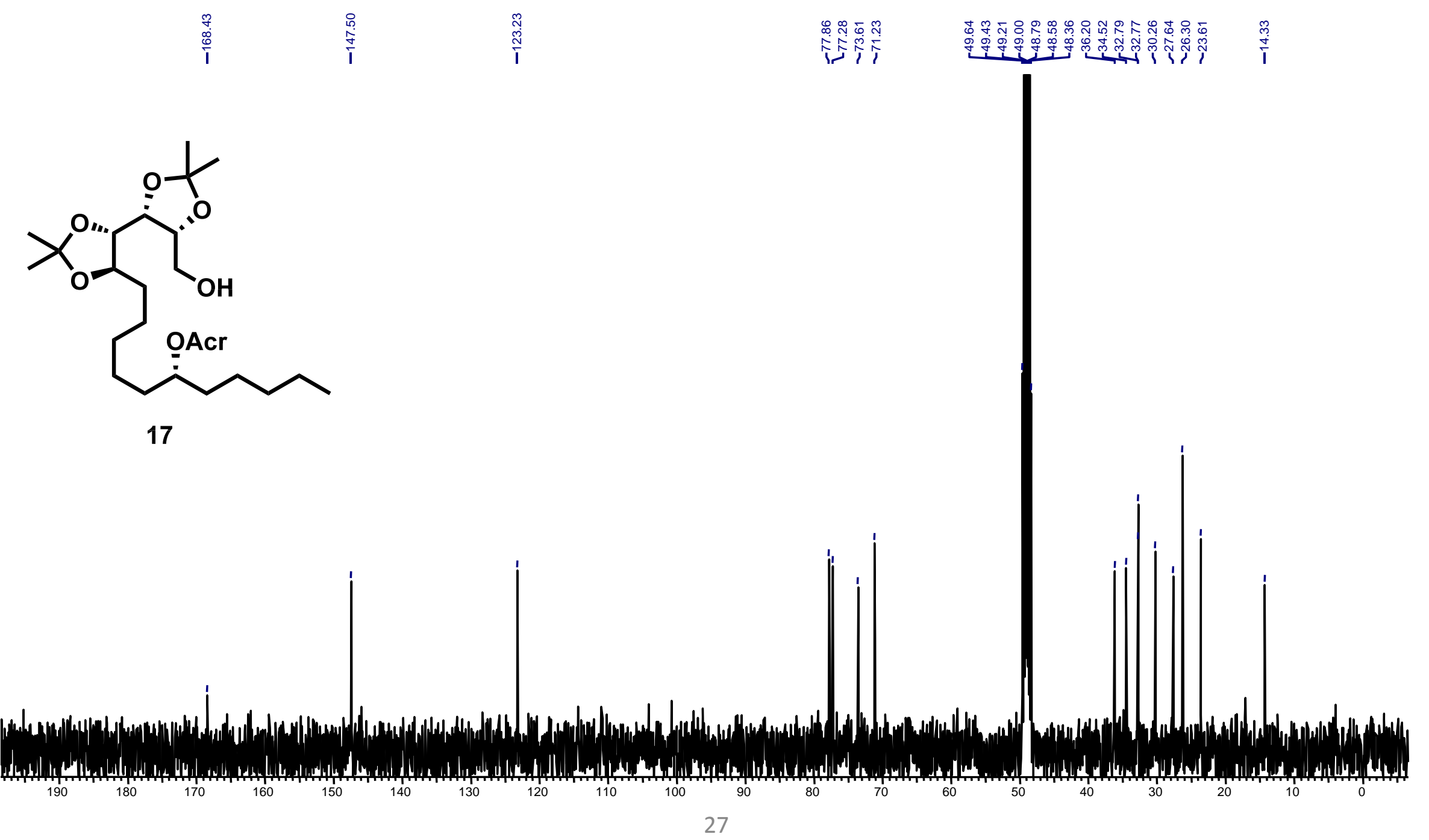


${ }^{1} \mathrm{H}$ NMR spectrum of compound $18\left(\mathrm{CDCl}_{3}, 400 \mathrm{MHz}\right)$

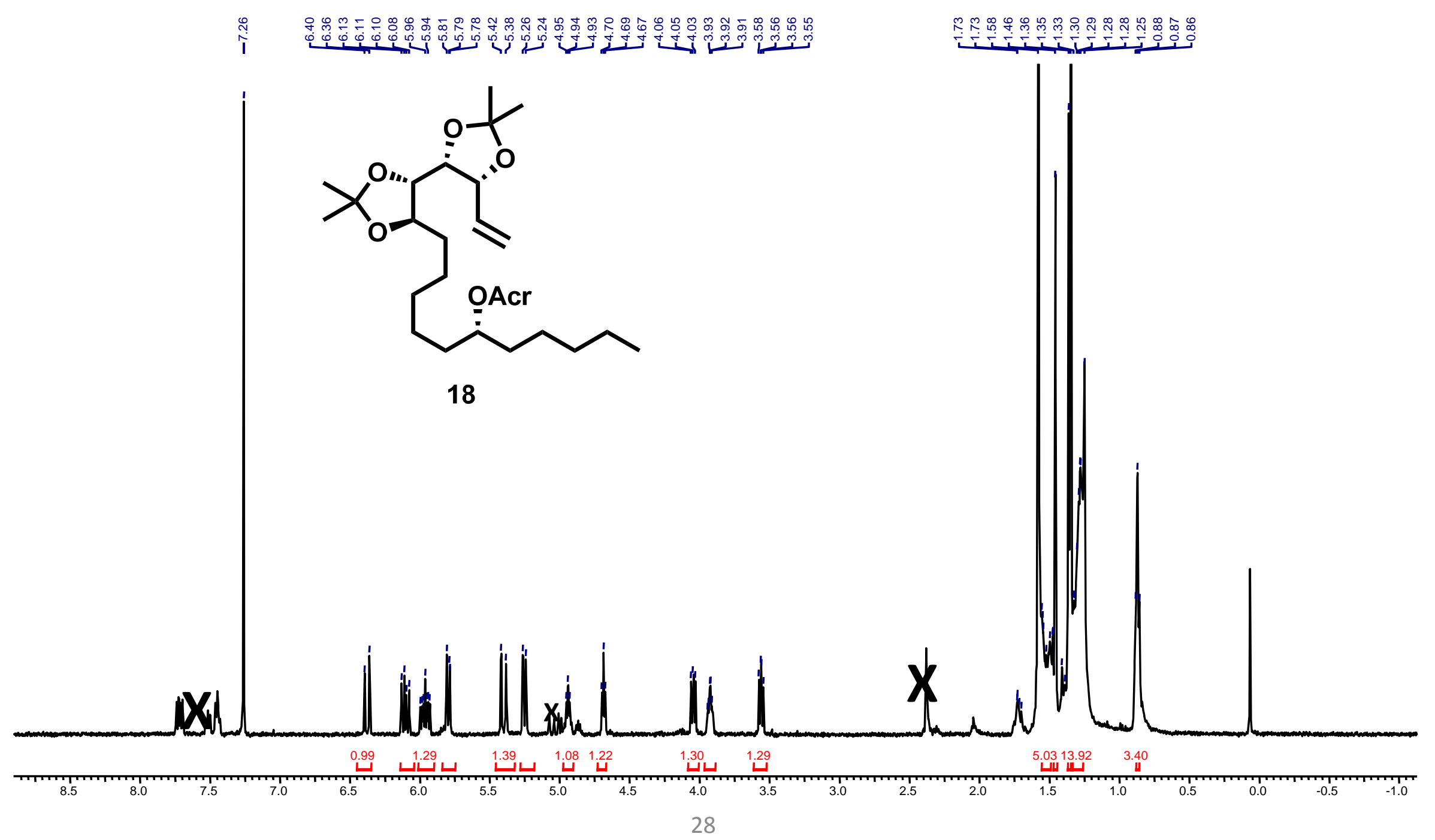


${ }^{13} \mathrm{C}$ NMR spectrum of compound $18\left(\mathrm{CDCl}_{3}, 100 \mathrm{MHz}\right)$

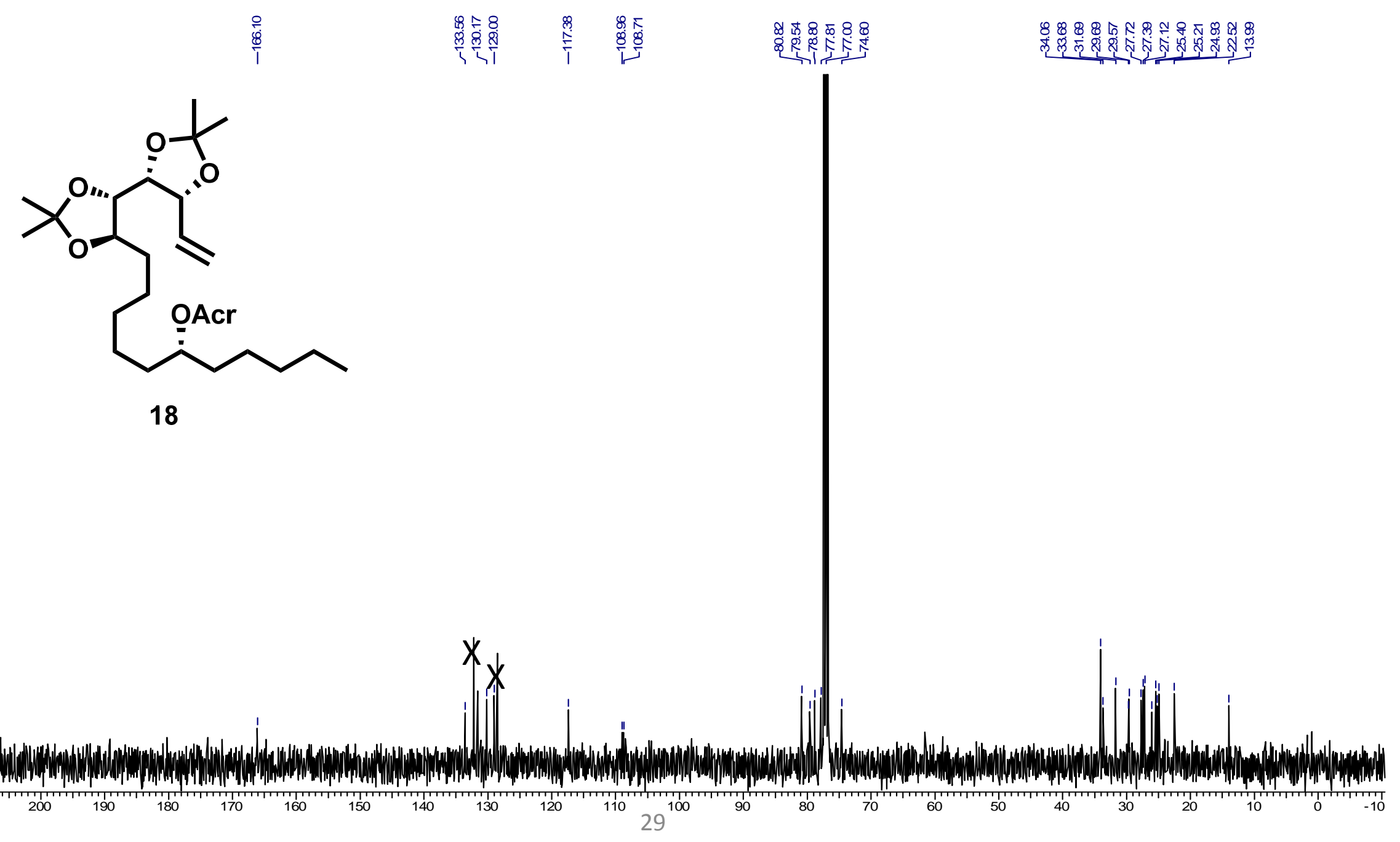




\section{${ }^{1} \mathrm{H}$ NMR spectrum of compound $19\left(\mathrm{CDCl}_{3}, 400 \mathrm{MHz}\right)$}

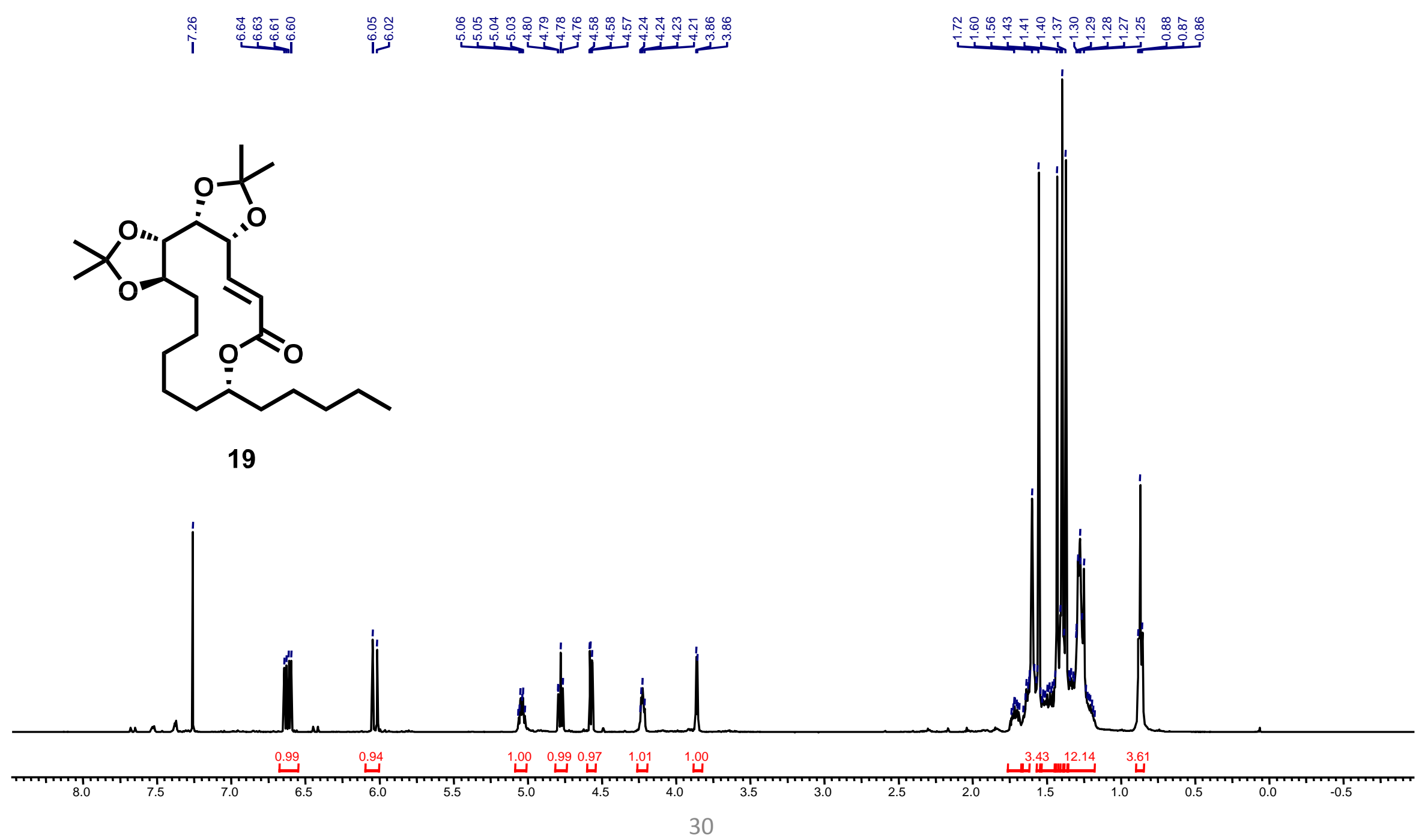


${ }^{13} \mathrm{C}$ NMR spectrum of compound $19\left(\mathrm{CDCl}_{3}, 100 \mathrm{MHz}\right)$

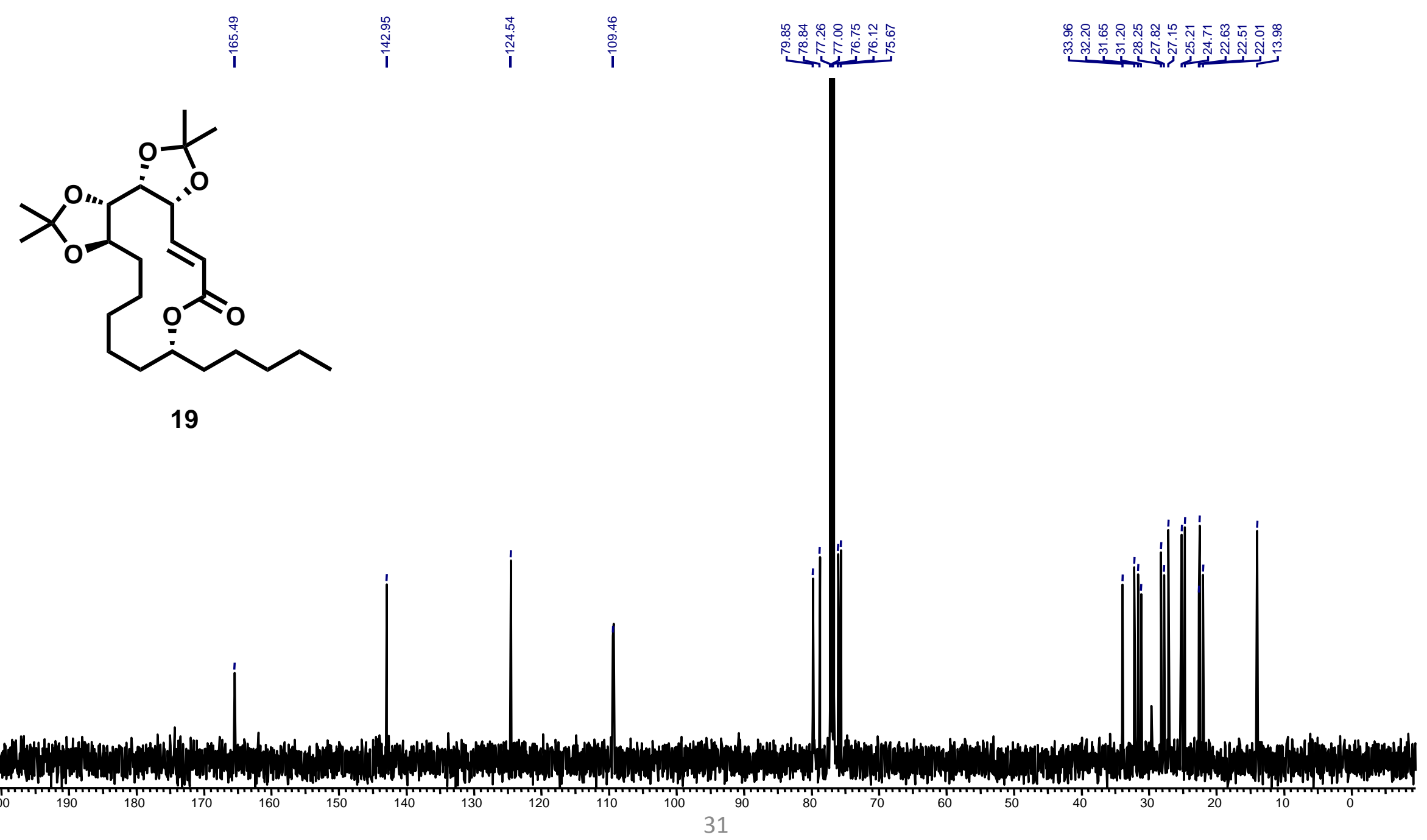




\section{${ }^{1} \mathrm{H}$ NMR spectrum of compound $4\left(\mathrm{CD}_{3} \mathrm{OD}, 400 \mathrm{MHz}\right)$}

Methanol; Layer No.: 1; Created by: Administrator; Created at: 09.08.2015 11:15:20 (GMT05:30)
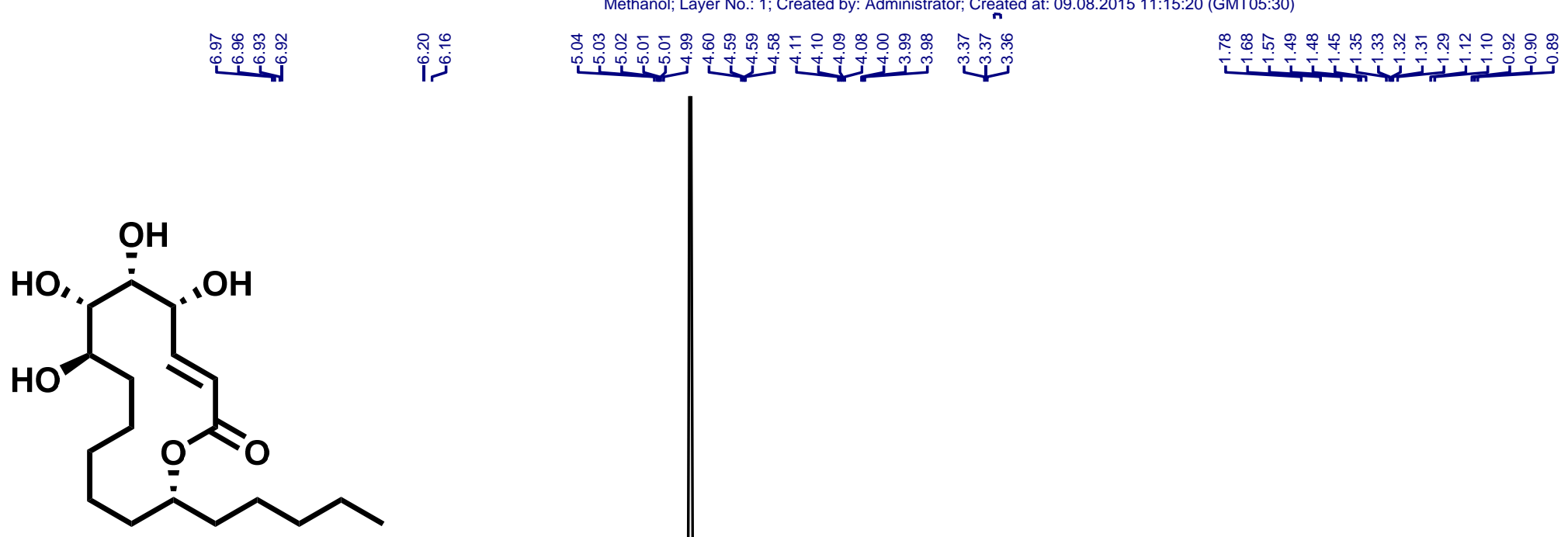

Gliomasolide C, 4

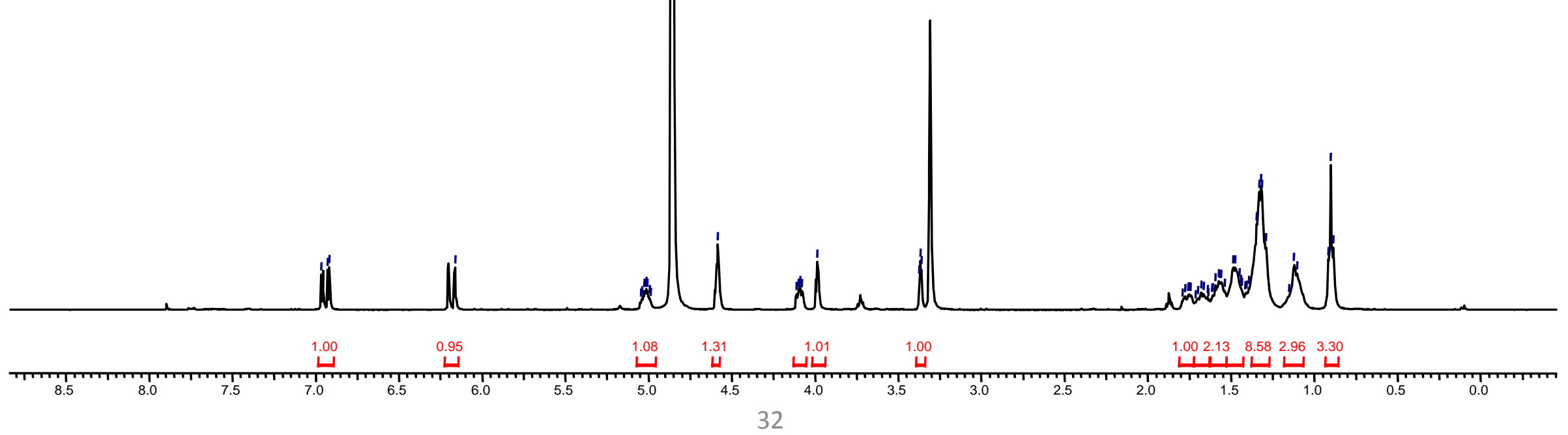




\section{${ }^{13} \mathrm{C}$ NMR spectrum of compound $4\left(\mathrm{CD}_{3} \mathrm{OD}, 100 \mathrm{MHz}\right)$}

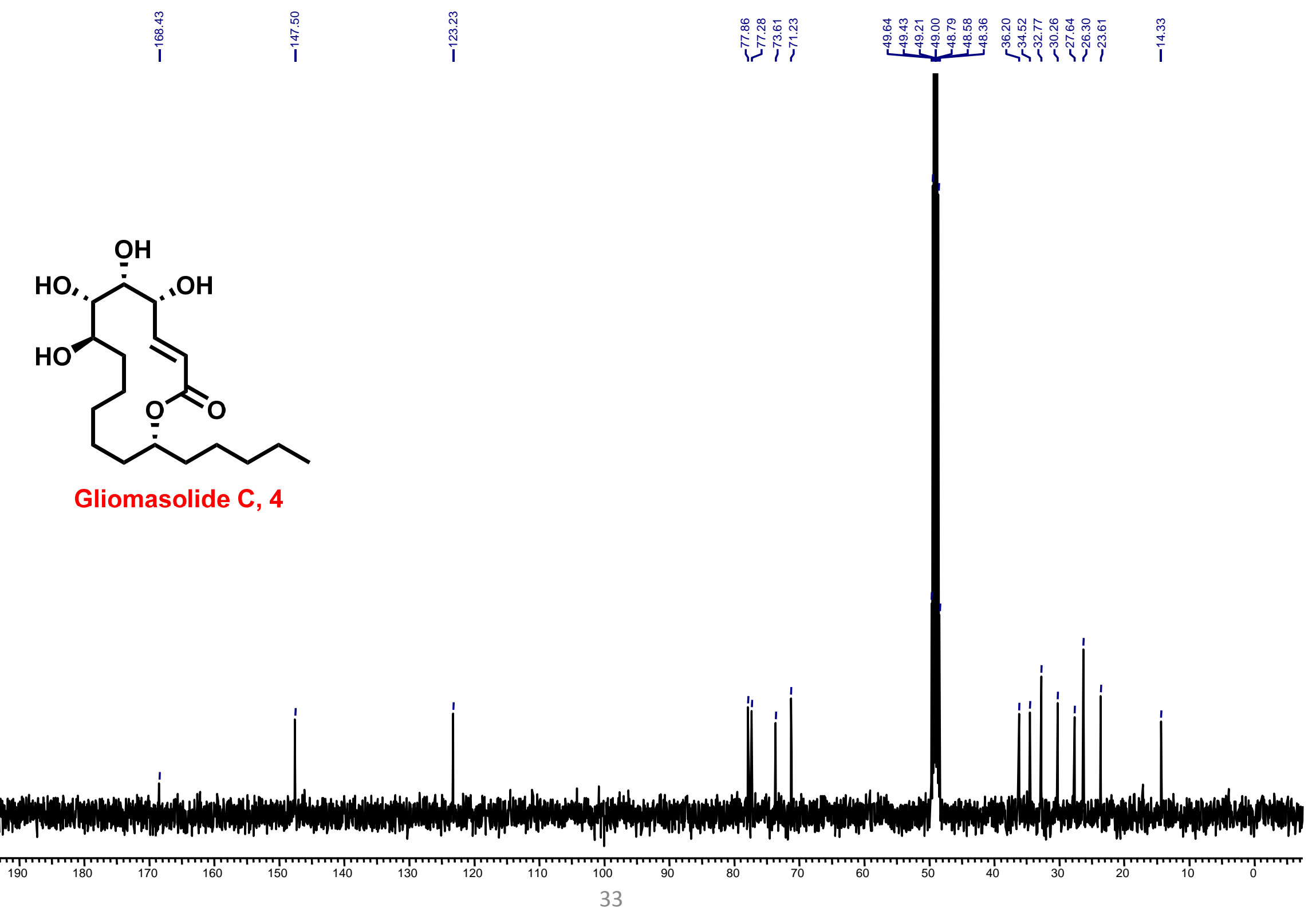




\section{${ }^{1} \mathrm{H}$ NMR spectrum of compound $20\left(\mathrm{CDCl}_{3}, 400 \mathrm{MHz}\right)$}

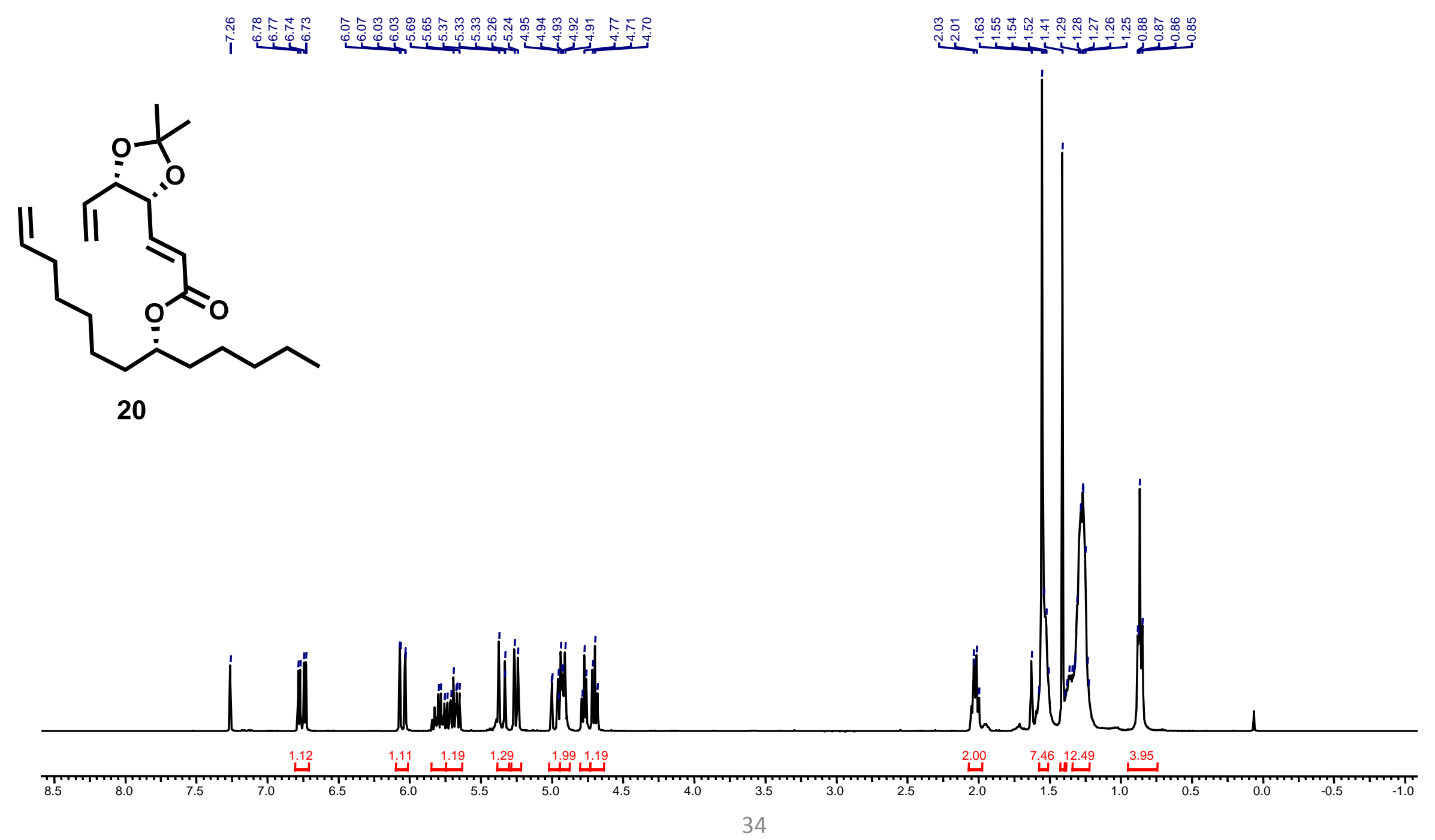


${ }^{13} \mathrm{C}$ NMR spectrum of compound $20\left(\mathrm{CDCl}_{3}, 100 \mathrm{MHz}\right)$

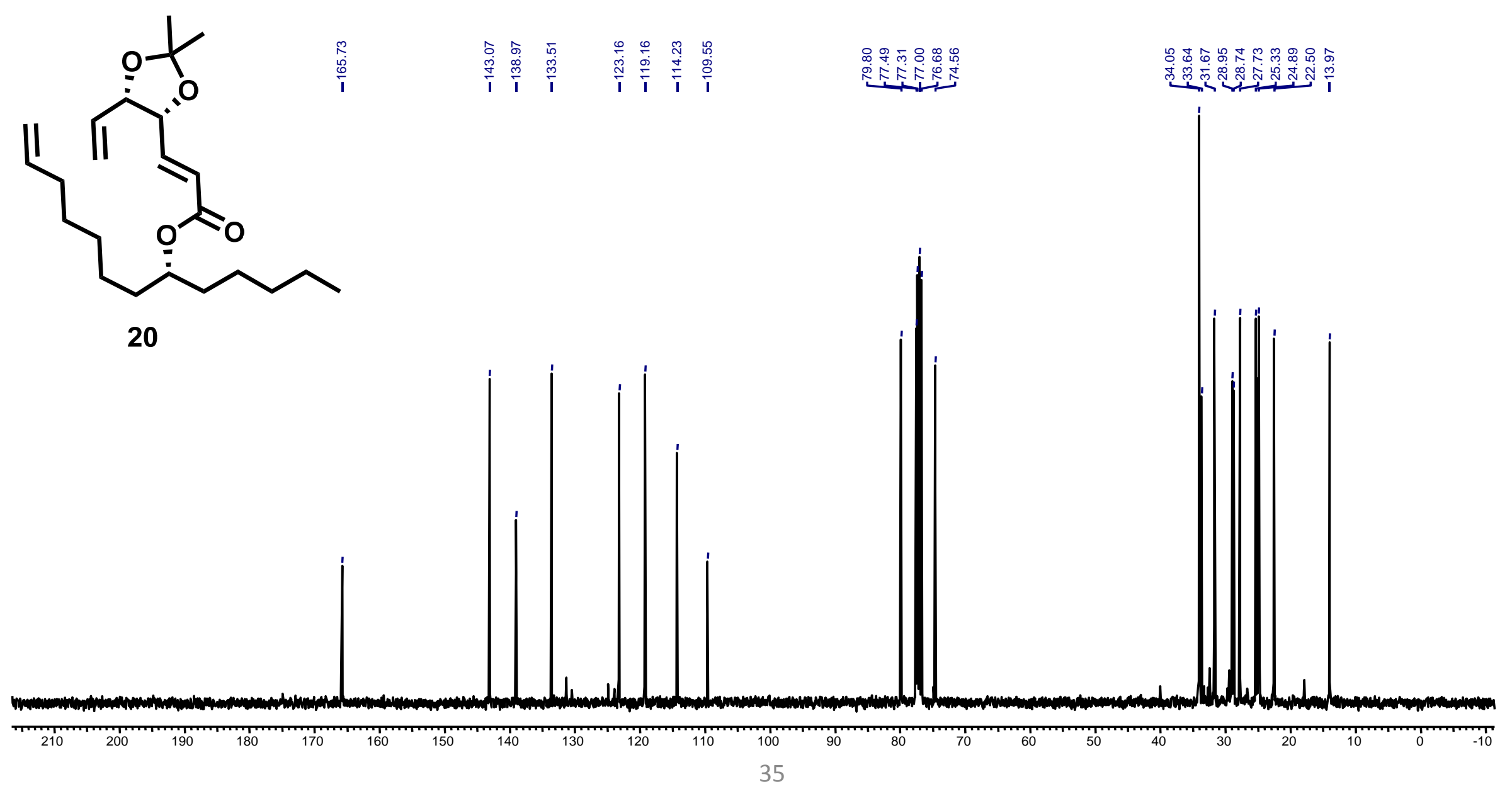




\section{${ }^{1} \mathrm{H}$ NMR spectrum of compound $21\left(\mathrm{CDCl}_{3}, 400 \mathrm{MHz}\right)$}

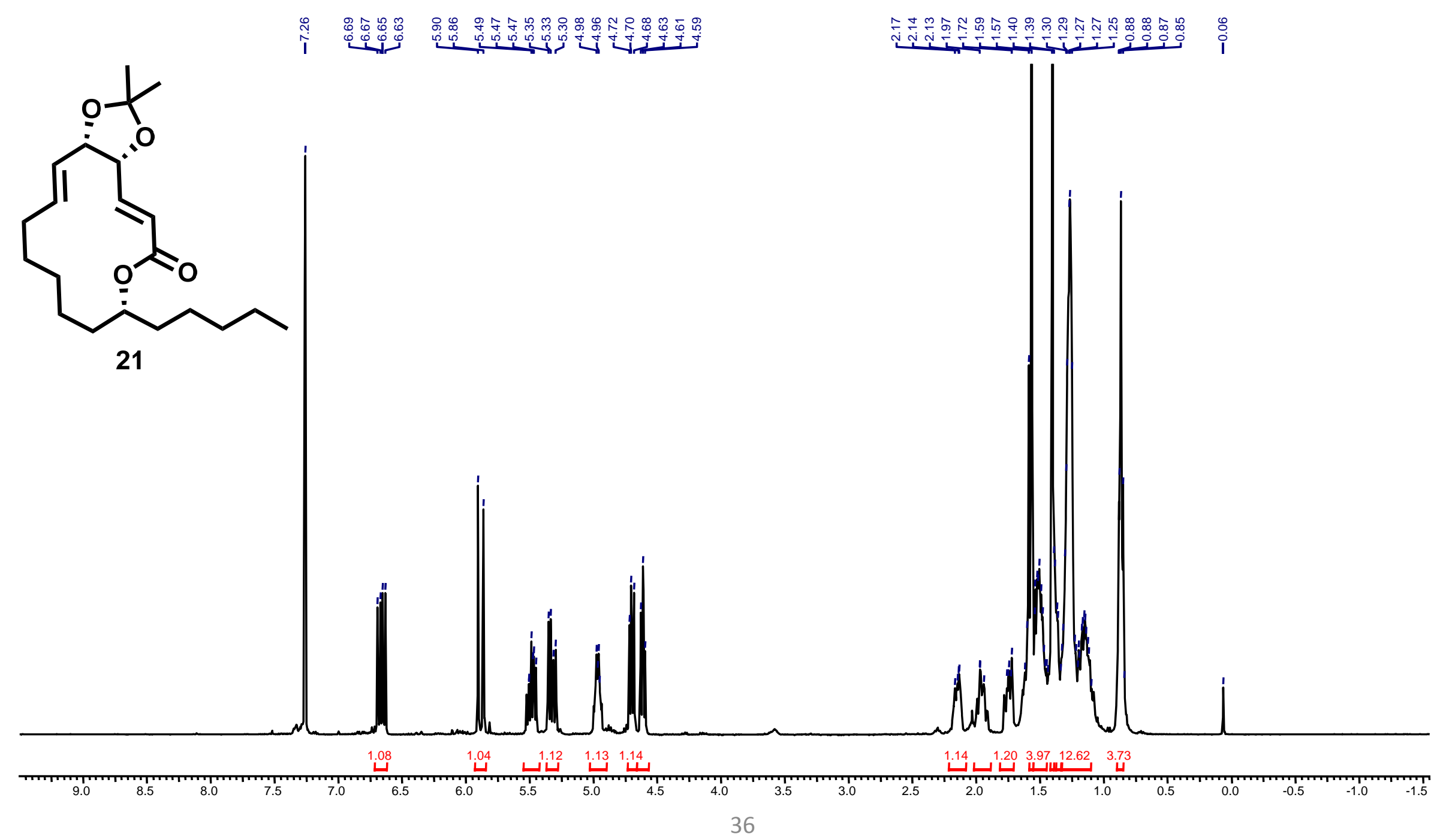


${ }^{13} \mathrm{C}$ NMR spectrum of compound $21\left(\mathrm{CDCl}_{3}, 100 \mathrm{MHz}\right)$
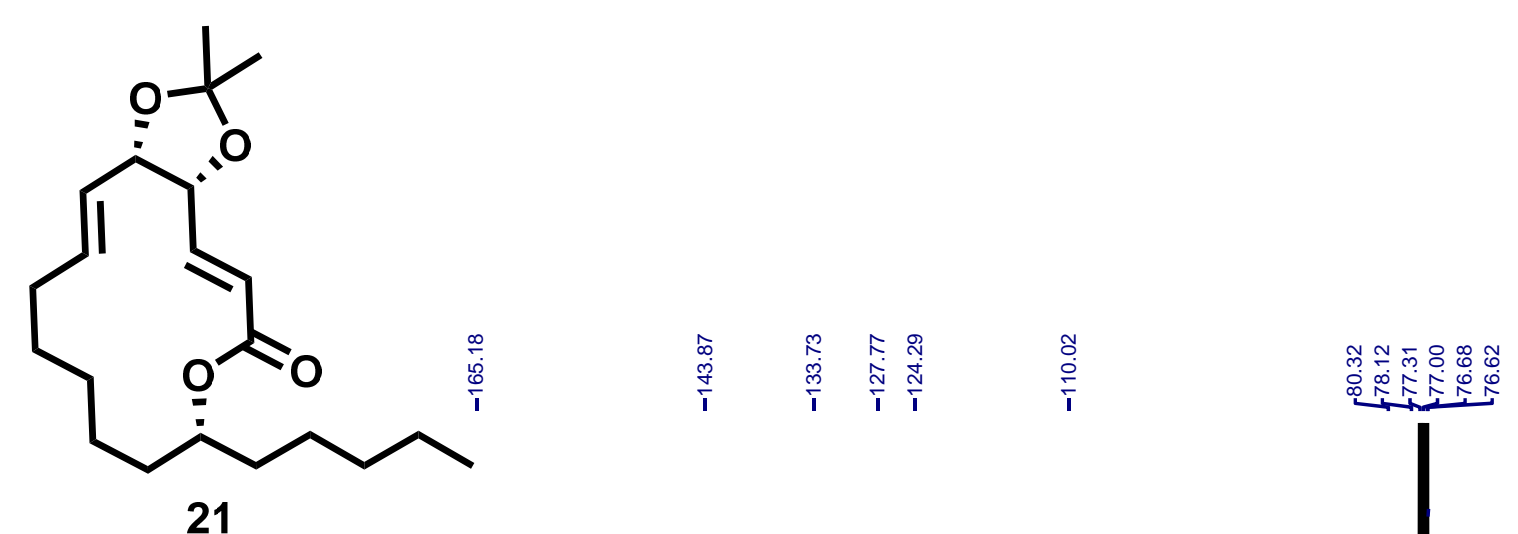

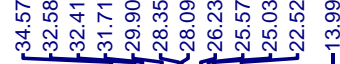

21

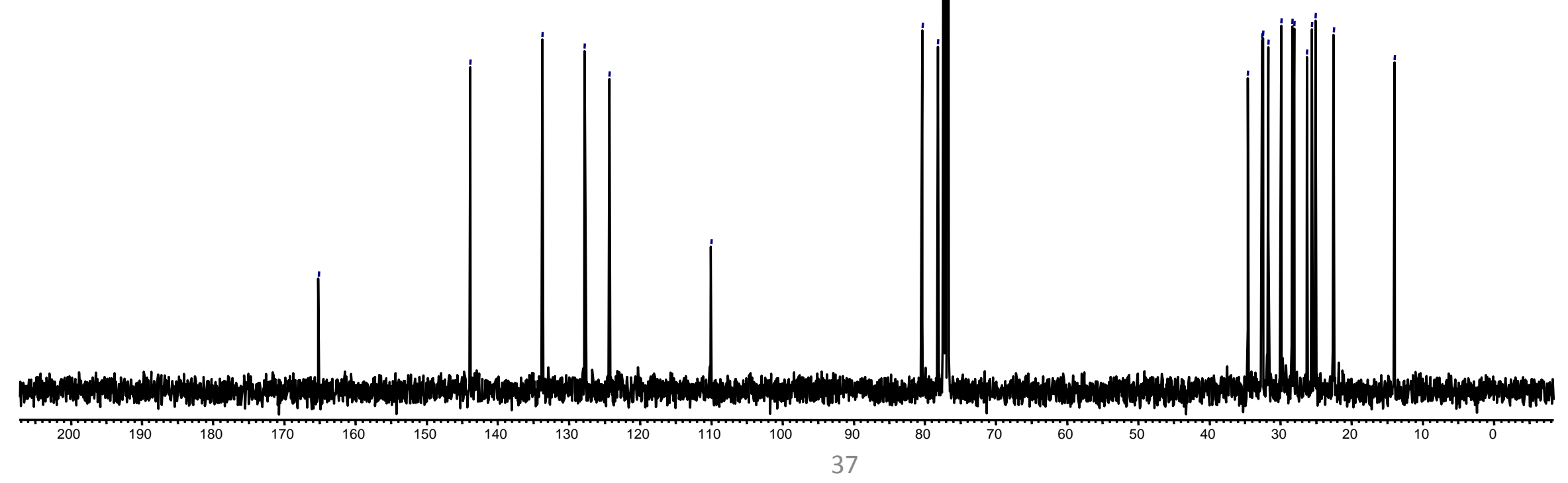

